\title{
ROBUST EXPONENTIAL DECAY OF CORRELATIONS FOR SINGULAR-FLOWS
}

\author{
VÍTOR ARAÚJO AND PAULO VARANDAS
}

\begin{abstract}
We construct open sets of $C^{k}(k \geq 2)$ vector fields with singularities that have robust exponential decay of correlations with respect to the unique physical measure. In particular we prove that the geometric Lorenz attractor has exponential decay of correlations with respect to the unique physical measure.
\end{abstract}

\section{Contents}

1. Introduction

2. Setting and statement of results

2.1. Uniformly expanding Markov map

2.2. Hyperbolic skew-product structure

2.3. Good hyperbolic skew-product semiflow

2.4. Statement of results

2.5. Strategy of the proof

Acknowledgements

3. The geometric Lorenz flow with smooth Lorenz map

3.1. Near the singularity

3.2. The rotating effect

3.3. The first return map to $S$

3.4. Properties of the one-dimensional map $f$

3.5. Properties of the map $g$

3.6. Persistence and smoothness of the contracting foliation 13

3.7. Robust transitivity properties 14

4. The geometric Lorenz flow is a good hyperbolic skew-product 16

4.1. The uniformly expanding Markov map 16

4.2. The good roof function 20

4.3. The hyperbolic skew-product structure 23

Date: November 5, 2018.

1991 Mathematics Subject Classification. 37D30, 37A25, 37C10 .

Key words and phrases. robust exponential decay of correlations, geometric Lorenz flow, uniform non integrability, induced uniformly expanding Markov map, hyperbolic skew-product semiflow, smooth stable foliation.

Part of this work was done during P.V. postdoctoral period at UFRJ-Rio de Janeiro with the finantial support of FAPERJ (Brazil-Rio de Janeiro). P.V was partially supported by FAPESB. V.A. was partially supported by CNPq, PRONEX-Dyn.Syst. and FAPERJ. 


\section{INTRODUCTION}

The ongoing interest in the mixing properties of deterministic dynamical systems was strongly inspired by the relevance of the subject in statistical mechanics. Moreover, the mixing properties of any given equilibrium state usually require deep knowledge of the system's chaotic features given for instance by its Lyapunov exponents.

Thermodynamical formalism was brought into the realm of dynamical systems by works of Sinai, Ruelle and Bowen [45, 12, 43]. In fact, since uniformly hyperbolic maps are semiconjugated to subshifts of finite type (via Markov partitions), any transitive uniformly hyperbolic map has a unique equilibrium state $\mu_{\phi}$ for every Hölder continuous potential $\phi$ with good mixing conditions: the correlation function

$$
C_{n}(\varphi, \psi)=\left|\int \varphi \cdot\left(\psi \circ f^{n}\right) d \mu_{\phi}-\int \varphi d \mu_{\phi} \int \psi d \mu_{\phi}\right|
$$

decays exponentially fast among Hölder continuous observables. Roughly, the robust chaotic features of uniformly hyperbolic dynamics are responsible by the very strong mixing properties. An extension of the thermodynamical formalism for uniformly hyperbolic (Axiom A) flows was also possible by the construction of finite Markov partitions obtained by Bowen and Ruelle in [13].

Even though uniformly hyperbolic (Axiom A) flows are semi-conjugated to suspension semiflows over subshifts of finite type, the mixing properties for time-continuous dynamical systems turned out to be much more subtle than the discrete time setting. On the one hand, hyperbolic suspension flows by a constant roof function are never topologically mixing despite the exponential mixing rate of the base transformation. On the other hand, the general feeling that topologically mixing uniformly hyperbolic flows should enjoy exponential decay of correlations was promptly put down by the counterexample of Ruelle [44, showing that there are topologically mixing Axiom A flows without exponential decay of correlations. In fact, examples of hyperbolic flows with arbitrary slow rate of decay of correlations were given by Pollicott [36]. Hence, despite several recent contributions, a complete understanding of the mixing properties for uniformly hyperbolic flows is still far from complete.

For a more detailed description of the state of the art let us mention that the proof of exponential decay of correlations for geodesic flows on manifolds of constant negative curvature was first obtained in two dimensions by Collet-Epstein-Gallavotti [16] and then in three dimensions by Pollicott [37] through group theoretical arguments.

Much more recently, Chernov [15] and Dolgopyat [18] studied Anosov flows and Liverani [28] extended such results for contact flows. Still in the uniformly hyperbolic context, Pollicott [38] extended the results in [18] to study the decay rate of equilibrium states associated to Hölder continuous potentials and Anosov flows. We should also refer that 
Dolgopyat [19] proved that typical Axiom A flows (in a probabilistic sense) have superpolynomial decay of correlations. In a more recent contribution, Field, Melbourne and Törok [21] proved that in fact a $C^{2}$-open and $C^{\infty}$-dense set of Axiom A flows have superpolynomial decay of correlations. Hence, Axiom A flows have robust fast mixing. This raises the following natural question.

Question 1. Is there some hyperbolic (non-singular) flow with robust exponential decay of correlations?

Rather surprisingly, there are some recent evidences that the presence of singularities appears as one important mechanism to obtain topologically and measure theoretical mixing. Roughly, orbits that approach singularities accelerate differently causing displacements of different order in the flow direction.

One of the most emblematic examples of flows where singular and regular behavior coexist are Lorenz attractors. In the mid seventies Afraimovich, Bykov and Shil'nikov [1] and Guckenheimer and Williams [24] introduced independently the geometric Lorenz attractors to model the original Lorenz attractors. The rigorous proof for the existence of the non-uniform hyperbolic Lorenz attractor for the parameter values originally suggested by E. Lorenz was obtained by Tucker [46] in the end of the 1990's. In addition, there was a general feeling that the Lorenz attractor should have a unique physical measure with exponential decay of correlations.

In more recent works there were several advances in that direction. First Luzzatto, Melbourne, Paccaut [30] proved that the geometric models for the Lorenz attractor are topologically mixing with respect to the unique physical measure. Then Araújo, Pacífico, Pujals, Viana [8] guarantee that every singular hyperbolic attractor (a class which contains the Lorenz and geometrical Lorenz attractors) carries a unique physical measure whose basin of attraction covers Lebesgue almost every point. Recently, in [22, 5], exponential decay of correlation for the Poincaré return map to suitably chosen cross-sections of geometric Lorenz flows and for the general case of singular-hyperbolic flows has been obtained. However the question about the exponential decay rate of the flow on this class of attractors remained open.

More recent developments include a criterium given by Baladi, Vallée [10] and Ávila, Goüezel, Yoccoz [9] to deduce exponential decay of correlations for suspension flows over $C^{2}$ Markov maps. The fact that here Markov partition may admit countably many elements implies that those classes of systems contain many important examples in non-uniformly hyperbolic dynamics as the suspension semiflows of the Maneville-Pommeau map, the Hénon maps, and other classes of flows as singular-hyperbolic attractors (e.g. the Lorenz or geometrical Lorenz attractor). A similar approach was pursued in [14] by Bufetov to obtain streched exponential decay of correlations for the Teichmüller flow on the space of Abelian differentials.

Our purpose here is to contribute to the ergodic theory of singular flows and to construct a nonempty open subset of singular flows with exponential decay of correlations. Let us mention that Question 1 is not answered in the uniformly hyperbolic context. Such class 
of flows, including the geometric Lorenz attractor, combine hyperbolic behavior with the existence of singularities.

Our strategy is to construct Lorenz attractors whose associated one-dimensional piecewise expanding transformation is twice differentiable and, hence, admits a countable Markov partition as in the above setting. Then, we prove that these flows are conjugated to suspension flows over a base with an hyperbolic structure and such that the height function satisfies a uniform non-integrability condition as introduced by Dolgopyat. Then we use a criteria from [10, 9] to deduce that such flows have exponential decay of correlations. Moreover, using [32, 27] we are also able to prove that these flows satisfy the central limit theorem.

Two final comments are in order. First let us mention that the Lorenz attractors associated to the original parameters obtained by E. Lorenz in 29] do not verify our assumption, and so the question of exponential decay of correlations for the original Lorenz attractor and small perturbations of it remains open. The second comment is that we expect that a similar approach may be applied to other equilibrium states.

Question 2. Do the equilibrium states constructed by Pacifico and Todd [35] for the contracting Lorenz attractor have exponential decay of correlations?

We finish with the following conjectures on the decay of correlations for general robustly transitive flows.

Conjecture 1. Non-hyperbolic robustly mixing flows in three-dimensional manifolds have robust exponential decay of correlations.

It is known that robustly transitive flows in dimension three are singular-hyperbolic, that is, are partially hyperbolic with one-dimensional contracting direction and a twodimensional volume expanding direction; see e.g. 34]. Moreover, if there are no singularities then the flow is uniformly hyperbolic and the decay of correlations of the SRB measure can be arbitrarily slow. See e.g. [7] for a rather complete description of the state of the art. However, it is not yet known wether all singular-hyperbolic flows are topologically mixing. Therefore the previous conjecture states that singularities are a mechanism to generate robust exponential decay of correlations in dimension three. Indeed we believe that it is possible to remove the regularity assumption.

Conjecture 2. $C^{1+\alpha}$ Lorenz attractors have robust exponential decay of correlations.

The reduction to a suspension semiflow over a non-uniformly expanding base transformation can also be performed in a higher dimensional class of examples; see [11]. These are just a particular example of a sectional-hyperbolic attractor; see [33]. The notion of sectionalhyperbolicity generalizes the notion of hyperbolicity for singular flows in any dimension and contains, in particular, the class of singular hyperbolic attractors in 3-manifolds.

Conjecture 3. Smooth singular flows in higher dimensions which are sectionally-hyperbolic exhibit robust exponential decay of correlations.

We believe our main result and its proof can be adapted to establish limit theorems for the distribution of random variables generated by the geometric Lorenz system. On the 
one hand, in a recent work, Holland and Melbourne [27] used that the geometric Lorenz attractor is a suspension flow to prove that all Lorenz attractors satisfy the central limit theorem and invariance principles, where no condition on the speed of decay of correlations was necessary. On the other hand, limit theorems for diffeomorphisms given as time- $t$ maps of flows are harder to obtain. Notice that even for uniformly hyperbolic flows the time-one maps are partially hyperbolic diffeomorphisms. A very general result was obtained by Melbourne and Törok [31] under some assumptions on the decay of correlations for the flow. We expect these ideas can be adapted to prove that the strong mixing properties for the $C^{2}$-open subset of geometric Lorenz attractors imply (robust) limit theorems for the corresponding time-one maps. More precisely we pose the following:

Conjecture 4. Let $\mathcal{U} \subset \mathfrak{X}^{s}(M)$ be the open family of vector fields for which we prove exponential decay of correlations, and denote by $\left(X_{t}\right)_{t}$ the flow generated by $X \in \mathcal{U}$. For all but countably many values of $t \in \mathbb{R}$ the time-t map $X_{t}$ the following Central Limit Theorem holds: for any $\varphi: \Delta_{r} \rightarrow \mathbb{R}$ in $L^{\infty}\left(\Delta_{r}\right)$ there exists $\sigma=\sigma(\varphi)>0$ such that

$$
\frac{1}{\sigma \sqrt{n}}\left[\sum_{j=0}^{n-1} \varphi\left(X_{t n}\right)-\int \varphi d \mu\right] \stackrel{\mathcal{D}}{\rightarrow} \mathcal{N}(0,1) .
$$

Exploring the same ideas from [31, we believe an Almost Sure Invariance Principle can also be obtained for the physical measure of this open set of geometric Lorenz flows with respect to the time-1 map.

The paper is organized as follows. In Section 2 we introduce some preliminary definitions and give the precise statements of our main results. In Section 3 we construct $C^{2}$-open sets of Lorenz attractors with smooth Lorenz one dimensional transformation. Finally, we prove that these attractors are conjugated to suspension semiflows with a good hyperbolic structure and, hence, have exponential decay of correlations.

\section{Setting And Statement of Results}

Throughout, let $M$ be a compact Riemannian manifold, let $d$ denote the induced Riemannian distance in $M,\|\cdot\|$ the Riemannian norm and Leb the induced normalized Riemannian volume form.

We will introduce the setting of induced maps and some concepts from the thermodynamical formalism of suspension semiflows. Our main results will be stated by the end of the section. In what follows we write $\|\cdot\|_{0}$ for the sup-norm in various functional spaces.

2.1. Uniformly expanding Markov map. We assume that $\cup_{\ell \in L} \Delta^{(\ell)}$ is an at most countable partition (Lebesgue modulo zero) of an open domain $\Delta \subset M$ by open subsets and let $F: \cup_{\ell \in L} \Delta^{(\ell)} \rightarrow \Delta$ be a $C^{r}$ uniformly expanding Markov map, $r \geq 2$, that is

(1) $F: \Delta^{\ell} \rightarrow \Delta$ is a $C^{r}$ diffeomorphism for every $\ell$;

(2) there are $C>0$ and $0<\lambda<1$ such that

(a) for every inverse branch $h_{n}$ of $F^{n}$, with $n \geq 1, d\left(h_{n}(x), h_{n}(y)\right) \leq C \lambda^{n} d(x, y)$; 
(b) if $J F$ is the Jacobian of $F$ with respect to the Lebesgue measure, then $\log J F$ is a $C^{1}$ function and $\sup |D((\log J F) \circ h)| \leq C$ for every inverse branch $h$ of $F$.

We denote by $\mathcal{H}_{n}$ the family of inverse branches of $F^{n}$. In many applications we have that $\Delta$ is a finite dimensional topological disk. It is well known that $F$ admits an invariant probability measure $\nu$ which is absolutely continuous with respect to Lebesgue.

2.2. Hyperbolic skew-product structure. We recall some notions previously used by [10] and [9]. We say that the roof function $r: \Delta \rightarrow \mathbb{R}^{+}$has exponential tail if there exists $\sigma_{0}>0$ such that $\int e^{\sigma_{0} r} d \nu<\infty$.

Definition 2.1. We say that the roof function $r$ is good if

(1) $r$ is bounded from below by some positive constant $r_{0}$;

(2) there exists $C>0$ such that $\sup _{h \in \mathcal{H}_{1}}\|D(r \circ h)\|_{0} \leq C<\infty$;

(3) it is not possible to write $r=v+u \circ F-u$ on $\Delta$, where $v: \Delta \rightarrow \mathbb{R}$ is constant on each $\Delta^{\ell}$ and $u: \Delta \rightarrow \mathbb{R}$ is a $C^{1}$-function.

Remark 2.2. The last condition in Definition 2.1 above corresponds to the uniform nonintegrability, or aperiodicity, condition defined by Baladi-Vallée in [10]. There are a number of equivalent conditions to this, as proved in [9, Proposition 7.5].

Now we define the hyperbolic skew-product structure with which Lorenz-like flows are endowed.

Definition 2.3. Let $F: \bigcup_{l} \Delta^{(l)} \rightarrow \Delta$ be a uniformly expanding Markov map, preserving an absolutely continuous probability measure $\nu$. A hyperbolic skew-product over $F$ is a map $\widehat{F}$ from a dense open subset of an open domain $\widehat{\Delta}$ of a compact Riemannian manifold $M$, to $\widehat{\Delta}$, satisfying the following properties:

(1) there exists a continuous map $\pi: \widehat{\Delta} \rightarrow \Delta$ such that $F \circ \pi=\pi \circ \widehat{F}$ whenever both members of the equality are defined;

(2) there is a $\widehat{F}$-invariant probability measure $\eta$ on $\widehat{\Delta}$, giving full mass to $\widehat{\Delta}$;

(3) there exists a family of probability measures $\left\{\eta_{x}\right\}_{x \in \Delta}$ on $\widehat{\Delta}$ which is a disintegration of $\eta$ over $\nu$, that is, $x \mapsto \eta_{x}$ is measurable, $\eta_{x}$ is supported on $\pi^{-1}(x)$ and, for each measurable subset $A$ of $\widehat{\Delta}$ we have $\eta(A)=\int \eta_{x}(A) d \nu(x)$. Moreover, this disintegration is smooth: we can find a constant $C>0$ such that, for any open subset $V \subset \cup \Delta^{(l)}$ and for each $u \in C^{1}\left(\pi^{-1}(V)\right)$, the function $\bar{u}: V \rightarrow \mathbb{R}, x \mapsto$ $\bar{u}(x):=\int u(y) d \eta_{x}(y)$ belongs to $C^{1}(V)$ and satisfies

$$
\sup _{x \in V}\|D \bar{u}(x)\| \leq C \sup _{y \in \pi^{-1}(V)}\|D u(y)\| .
$$

(4) there is $\kappa>1$ such that, for all $w_{1}, w_{2} \in \widehat{\Delta}$ in the same leaf, i.e. $\pi\left(w_{1}\right)=\pi\left(w_{2}\right)$, we have $d\left(\widehat{F} w_{1}, \widehat{F} w_{2}\right) \leq \kappa^{-1} d\left(w_{1}, w_{2}\right)$. 
2.3. Good hyperbolic skew-product semiflow. Now we introduce suspension semiflows over the class of dynamical systems presented above. Given a function $r: \cup_{\ell \in L} \Delta^{(\ell)} \rightarrow$ $\left[r_{0},+\infty\right)$ for some $r_{0}>0$ we define

$$
\widehat{\Delta}_{r}=\{(w, t): w \in \widehat{\Delta}, 0 \leq t \leq r(\pi(w))\} / \sim,
$$

where $\sim$ is an equivalence relation that identifies the pairs $(w, r(\pi(w)))$ and $(F(w), 0)$. For any $\ell \in L$ let $\widehat{\Delta}_{r}^{(\ell)}$ be defined accordingly using $\Delta_{r}^{(\ell)}$. In this way it is natural to consider the suspension semiflow $\left(Y_{t}\right)_{t}$ given by $Y_{t}(w, s)=(w, s+t)$. In these coordinates it coincides with the flow which consists in the displacement along the "vertical" direction. Moreover, we will say that $r$ is the roof function of the suspension skew-product semi-flow $\left(Y_{t}\right)_{t}$ over the map $\widehat{F}$.

If $Y_{t}$ is a semiflow over a hyperbolic skew-product with a good roof function which, moreover, has exponential tail, then we say that $Y_{t}$ is a good hyperbolic skew-product semiflow. If $\eta$ is an $\widehat{F}$-invariant probability measure so that $\int r d \eta<\infty$, then $\left(Y_{t}\right)_{t}$ preserves the probability measure $\bar{\eta}$ given by $\bar{\eta}=(\eta \otimes$ Leb $) / \int r d \eta$.

2.4. Statement of results. Let $C^{1}\left(\widehat{\Delta}_{r}\right)$ be the space of bounded observables $g: \widehat{\Delta}_{r} \rightarrow \mathbb{R}$ that are piecewise $C^{1}$ continuous (i.e. $C^{1}$ in each element $\widehat{\Delta}_{r}^{(\ell)}$ ) endowed with the norm $\|g\|_{1}:=\sup _{w \in \widehat{\Delta}}|g(w)|+\sup _{w \in \widehat{\Delta}}\|D g(w)\|$. The following was proved in [9, Theorem 2.7].

Theorem 2.4. Let $Y_{t}$ be a good hyperbolic skew-product semi-flow on a space $\widehat{\Delta}_{r}$, preserving the probability measure $\bar{\eta}$. There exist constants $C>0$ and $\delta>0$ such that, for each pair of functions $\varphi, \psi \in C^{1}\left(\widehat{\Delta}_{r}\right)$ and $t \geq 0$,

$$
\left|\int \varphi \cdot \psi \circ Y_{t} d \bar{\eta}-\left(\int \varphi d \bar{\eta}\right)\left(\int \psi d \bar{\eta}\right)\right| \leq C\|\varphi\|_{1}\|\psi\|_{1} e^{-\delta t} .
$$

The main arguments in this paper prove the following.

Theorem A. Given any compact 3-manifold $M$, for each $s \geq 2$ we can find an open subset $\mathcal{U}$ of $\mathfrak{X}^{s}(M)$ such that each $X \in \mathcal{U}$ exhibits a geometric Lorenz flow which is $C^{s}$-smoothly semi-conjugated to a good hyperbolic skew-product semi-flow.

The meaning of the smooth semi-conjugacy above is: if $U$ is an open neighborhood such that $\overline{X^{t}(U)} \subset U$ for all $t>0$ and the attractor is given by $\Lambda_{X}:=\cap_{t \geq 0} X^{t}(U)$, there exists a semi-flow $Y_{t}$ on $\widehat{\Delta}_{r}$ as stated above, together with a $C^{s}$ local diffeomorphism $\phi=\phi_{X}: \widehat{\Delta}_{r} \rightarrow M$, whose image contains an open neighborhood of the geometric Lorenz attractor $\Lambda_{X}$, for $X \in \mathcal{U}$, satisfying $\phi_{X} \circ Y_{t}=X_{t} \circ \phi_{X}$ at all points where both sides of the equality are defined. Moreover $\phi_{*} \bar{\eta}=\mu$, where $\mu$ is the physical measure supported on $\Lambda_{X}$, see e.g. [8, 7].

Corollary A. The geometric Lorenz attractors given in Theorem A have exponential decay of correlations for $C^{1}$ observables.

Indeed, if we take $\varphi, \psi$ a pair of $C^{1}$ functions on $\Lambda_{X}$, for some $X \in \mathcal{U}$, then these maps are restriction of $C^{1}$ maps on an open neighborhood $W$ of $\Lambda_{X}$ in $M$, which we denote by 
the same letters. We can assume that $W$ contains the image $\phi\left(\widehat{\Delta}_{r}\right)$ of $\phi$, for otherwise we can extend $\varphi, \psi$ to this neighborhood using bump functions without changing their values over $\Lambda_{X}$. Hence $\bar{\varphi}:=\varphi \circ \phi, \bar{\psi}:=\psi \circ \phi$ are $C^{1}$ functions on $\widehat{\Delta}_{r}$ and

$$
\begin{aligned}
\int \bar{\varphi} \cdot \bar{\psi} \circ Y_{t} d \bar{\eta} & =\int(\varphi \circ \phi) \cdot\left(\psi \circ \phi \circ Y_{t}\right) d \bar{\eta}=\int(\varphi \circ \phi) \cdot\left(\psi \circ X_{t} \circ \phi\right) d \bar{\eta} \\
& =\int \varphi \cdot\left(\psi \circ X_{t}\right) d\left(\phi_{*}(\bar{\eta})\right)=\int \varphi \cdot\left(\psi \circ X_{t}\right) d \mu,
\end{aligned}
$$

so the exponential decay of correlations follows from Theorem 2.4.

2.5. Strategy of the proof. The proof of the exponential decay of correlations for singular flows stated in Corollary $\mathrm{A}$ for the class of Lorenz attractors associated to vector fields in the open sets of Theorem $\mathrm{A}$ consists of three main steps.

First we prove that every three-dimensional manifold admit Lorenz-like attractors whose one-dimensional stable foliation is $C^{2}$. Moreover, such a contruction is robust in the sense that it holds for every $C^{2}$ close vector field. In fact we get that the restriction of the flows to the Lorenz-like attractors are smoothly semi-conjugated to suspension over a $C^{2}$ non-uniformly hyperbolic hyperbolic Poincaré map, that is, a partially hyperbolic transformation with one-dimensional stable direction and one-dimensional central direction with positive Lyapunov exponent with respect to an absolutely continuous invariant measure. The crucial regularity of the stable foliation is a consequence of a strong partially hyperbolicity of the Poincaré transformation requires a condition on the eigenvalues of the singularity, which is a $C^{1}$-open condition in a neighborhood of the original vector field.

The second ingredient is to prove that such flows are semi-conjugated to suspension flows whose roof function is constant along stable leaves. Such a property is obtained first for the geometric Lorenz attractor by construction. Although all sufficiently close vector fields still preserve the global cross-section for the original flow, it is most likely that the Poincaré return time to the original cross-section of those flows not to be constant along the stable leaves. So, for every sufficiently close flow we consider adapted sections, that is, sections that are foliated by the one-dimensional strong-stable leaves (see e.g. [8]). Hence, the invariance of the strong stable foliation guarantees that the first return time function is constant on stable leaves.

Finally we show that these flows satisfy the assumptions of [10, 9] on a criterium for exponential decay of correlations. Namely, the flow is conjugated to a suspension flow over a hyperbolic skew-product that admits a unique SRB measure with a smooth disintegration along the strong-stable foliation, and whose induced roof function satisfies a non-integrability condition and has exponential tail. For the later we revisit the construction of the SRB measure for Lorenz attractors and obtain a disintegration of the measure as fixed points associated to suitable transfer operators.

Acknowledgements. Part of this work was done during the stay of P.V. at the conference Low-Dimensional Dynamics as an activity of the CODY Autumn in Warsaw, whose excellent research conditions are greatly acknowledged. The authors are deeply grateful 
to the anonimous referee for many suggestions and comments that helped to improve the manuscript.

\section{The Geometric Lorenz Flow With Smooth Lorenz map}

Here we describe the construction of geometric Lorenz flows with $C^{k}$ smooth strongstable foliation, for each integer $k \geq 2$, following [7, Chap. 3, Sect. 3.3] and taking advantage of the idea of $k$-domination from [26]. The proof of the next proposition will be given along the rest of this section.

Proposition 3.1. Given $k \in \mathbb{Z}^{+}$there exists a $C^{k}$ vector field $X$ on $\mathbb{R}^{3}$ and a $C^{k}$ neighborhood $\mathcal{U}$ of $X$ in $\mathfrak{X}^{k}\left(\mathbb{R}^{3}\right)$ such that

- there exists a trapping region $U$ containing a surface cross-section $S$ for the flow of every $Y \in \mathcal{U}$;

- the maximal positively invariant subset $\Lambda_{Y}$ inside $U$ for the flow of $Y$ is a transitive attractor containing a hyperbolic singularity $\sigma$;

- the first return map $P_{Y}$ from $S^{*} \subset S$ to $S$ admits a $C^{k}$ smooth uniformly contracting foliation $\mathcal{F}_{Y}$, where $S^{*}=S \backslash W_{l o c}^{s}(\sigma)$;

- the induced one-dimensional quotient map $f_{Y}=P_{Y} / \mathcal{F}_{Y}$ is a piecewise $C^{k}$ smooth expanding map with two branches defined on intervals $I^{ \pm}$, where $\left|D f_{Y}\right|>\sqrt{2}$ having a common boundary point 0 , in a neighborhood of which the derivative $D f_{Y}$ grows as the logarithm of the distance to 0 ;

- the map $f_{Y}$ is locally eventually onto: every interval $J$ in the domain of $f_{Y}$ admits a subinterval $J_{0}$ and some iterate $n>2$ such that $f^{n}\left(J_{0}\right)$ contains either $I^{-}$or $I^{+}$.

A similar idea in the flow setting was used in [42] to obtain a $C^{2}$ strong-stable foliation on a singular attractor.

3.1. Near the singularity. In a neighborhood of the origin we consider the linear system $(\dot{x}, \dot{y}, \dot{z})=\left(\lambda_{1} x, \lambda_{2} y, \lambda_{3} z\right)$, thus

$$
X^{t}\left(x_{0}, y_{0}, z_{0}\right)=\left(e^{\lambda_{1} t} x_{0}, e^{\lambda_{2} t} y_{0}, e^{\lambda_{3} t} z_{0}\right),
$$

where $\lambda_{2}<\lambda_{3}<0<-\lambda_{3}<\lambda_{1}$ and $\left(x_{0}, y_{0}, z_{0}\right) \in \mathbb{R}^{3}$ is an arbitrary initial point near the origin.

To ensure that arbitrarily small $C^{2}$ perturbation of this flow are still smoothly linearizable near the continuation of the hyperbolic singularity $\sigma$, we use a smooth linearization result which can be found in Hartman [25, Theorem 12.1, p. 257].

Theorem 3.2. Let $n \in \mathbb{Z}^{+}$be given. Then there exists an integer $N=N(n) \geq 2$ such that: if $\Gamma$ is a real non-singular $d \times d$ matrix with eigenvalues $\gamma_{1}, \ldots, \gamma_{d}$ satisfying

$$
\sum_{i=1}^{d} m_{i} \gamma_{i} \neq \gamma_{k} \quad \text { for all } \quad k=1, \ldots, d \quad \text { and } \quad 2 \leq \sum_{j=1}^{d} m_{j} \leq N
$$

and if $\dot{\xi}=\Gamma \xi+\Xi(\xi)$ and $\dot{\zeta}=\Gamma \zeta$, where $\xi, \zeta \in \mathbb{R}^{d}$ and $\Xi$ is of class $C^{N}$ for small $\|\xi\|$ with $\Xi(0)=0, \partial_{\xi} \Xi(0)=0$; then there exists a $C^{n}$ diffeomorphism $R$ from a neighborhood of 
$\xi=0$ to a neighborhood of $\zeta=0$ such that $R \xi_{t} R^{-1}=\zeta_{t}$ for all $t \in \mathbb{R}$ and initial conditions for which the flows $\zeta_{t}$ and $\xi_{t}$ are defined in the corresponding neighborhood of the origin.

Hence it is enough for us to choose the eigenvalues $\left(\lambda_{1}, \lambda_{2}, \lambda_{3}\right) \in \mathbb{R}^{3}$ satisfying a finite set of non-resonance relations (3) for a certain $N=N(2)$. For this condition defines an open set in $\mathbb{R}^{3}$ and so all small $C^{1}$ perturbations $Y$ of the vector field $X$ will have a singularity whose eigenvalues $\left(\lambda_{1}(Y), \lambda_{2}(Y), \lambda_{3}(Y)\right)$ are still in the $C^{2}$ linearizing region.

We consider the set $S=\{(x, y, 1):|x| \leq 1 / 2,|y| \leq 1 / 2\}$ and

$$
\begin{aligned}
& S^{-}=\{(x, y, 1) \in S: x<0\}, \\
& S^{+}=\{(x, y, 1) \in S: x>0\} \quad \text { and } \\
& S^{*}=S^{-} \cup S^{+}=S \backslash \Gamma, \quad \text { where } \quad \Gamma=\{(x, y, 1) \in S: x=0\} \text {. }
\end{aligned}
$$

We assume without loss of generality that $S$ is a transverse section to the flow so that every trajectory eventually crosses $S$ in the direction of the negative $z$ axis as in Figure 1. Note that $\Gamma$ is the intersection of $S$ with the local stable manifold $W_{l o c}^{s}(\sigma)$ of the equilibrium $\sigma=(0,0,0): S^{*}=S \backslash W_{l o c}^{s}(\sigma)$. Hence we get

$$
X^{\tau}\left(x_{0}, y_{0}, 1\right)=\left(\operatorname{sgn}\left(x_{0}\right), y_{0} e^{\lambda_{2} \tau\left(x_{0}\right)}, e^{\lambda_{3} \tau\left(x_{0}\right)}\right)=\left(\operatorname{sgn}\left(x_{0}\right), y_{0}\left|x_{0}\right|^{-\frac{\lambda_{2}}{\lambda_{1}}},\left|x_{0}\right|^{-\frac{\lambda_{3}}{\lambda_{1}}}\right)
$$

where $\operatorname{sgn}(x)=x /|x|$ for $x \neq 0$, and $0<\alpha=-\frac{\lambda_{3}}{\lambda_{1}}<1<\beta=-\frac{\lambda_{2}}{\lambda_{1}}$ by the choice of the eigenvalues.

Consider also $\Sigma=\{(x, y, z):|x|=1\}=\Sigma^{-} \cup \Sigma^{+}$with $\Sigma^{ \pm}=\{(x, y, z): x= \pm 1\}$. For each $\left(x_{0}, y_{0}, 1\right) \in S^{*}$ the time $\widetilde{\tau}$ such that $X^{\widetilde{\tau}}\left(x_{0}, y_{0}, 1\right) \in \Sigma$ is given by $\widetilde{\tau}\left(x_{0}\right)=-\frac{1}{\lambda_{1}} \log \left|x_{0}\right|$, which depends on $x_{0} \in S^{*}$ only and is integrable with respect to Lebesgue measure on any bounded interval $J$ of the real line: $0<\int_{J} \widetilde{\tau}\left(x_{0}\right) d \lambda\left(x_{0}\right)<\infty$; see [7, 35]. Moreover we also have that $\tilde{\tau}$ is bounded from below by $\tau_{0}:=\log 2 / \lambda_{1}>0$. Let $L: S^{*} \rightarrow \Sigma$ be given by

$$
L(x, y, 1)=\left(\operatorname{sgn}(x), y|x|^{\beta},|x|^{\alpha}\right) .
$$

Clearly each line segment $S^{*} \cap\left\{x=x_{0}\right\}$ is taken to another line segment $\Sigma \cap\left\{z=z_{0}\right\}$ as sketched in Figure 1.

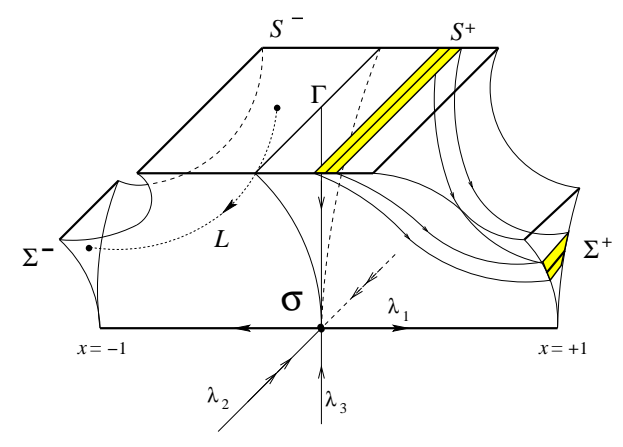

Figure 1. Behavior near the origin. 
3.2. The rotating effect. To imitate the random turns of a regular orbit around the origin and obtain a butterfly shape for our flow, as in the original Lorenz flow, the sets $\Sigma^{ \pm}$ should return to the cross-section $S$ through a flow described by a suitable composition of a rotation $R_{ \pm}$, an expansion $E_{ \pm \theta}$ and a translation $T_{ \pm}$.

We assume that the "triangles" $L\left(S^{ \pm}\right)$are compressed in the $y$-direction and stretched on the other transverse direction and that this return map takes line segments $\Sigma \cap\left\{z=z_{0}\right\}$ into line segments $S \cap\left\{x=x_{1}\right\}$, as sketched in Figure 2.

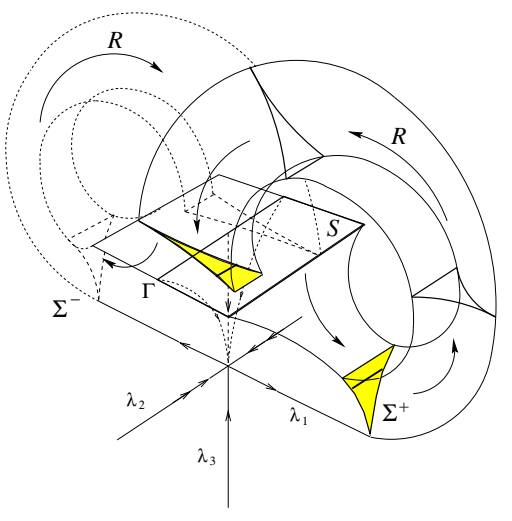

Figure 2. $R$ takes $\Sigma^{ \pm}$to $S$.

The choice of $R_{ \pm}, T_{ \pm}, E_{ \pm \theta}$ can be seen in [7, Chapter 3, Section 3] of [22].

These transformations $R_{ \pm}, E_{ \pm \theta}, T_{ \pm}$take line segments $\Sigma^{ \pm} \cap\left\{z=z_{0}\right\}$ into line segments $S \cap\left\{x=x_{1}\right\}$ as shown in Figure 2, and so does the composition $T_{ \pm} \circ E_{ \pm \theta} \circ R_{ \pm}$.

This composition of linear maps describes a vector field in a region outside $[-1,1]^{3}$, in the sense that one can use the above linear maps to define a vector field $X$ such that the first return map to $S$ of the associated flow realizes $T_{ \pm} \circ E_{ \pm \theta} \circ R_{ \pm}$as a map $\Sigma^{ \pm} \rightarrow S$.

We note that the flow on the attractor we are constructing will pass through the region between $\Sigma^{ \pm}$and $S$ in a relatively small time with respect the the linearized region. The linearized regions will then dominate all estimates of expansion/contraction.

More precisely, the time a point $\left(x_{0}, y_{0}, 1\right) \in S^{*}$ takes to return to $S$ is given by $\tau_{X}\left(x_{0}, y_{0}\right)=\tau\left(x_{0}\right)=\widetilde{\tau}\left(x_{0}\right)+s_{0}=-\left(1 / \lambda_{1}\right) \log \left|x_{0}\right|+s_{0}$, where $s_{0}>0$ is the constant time "lateral triangles" $\Sigma^{ \pm}$take to flow until $S$. Hence the return time to $S$ is clearly dominated by the behavior of $\widetilde{\tau}$ and the behavior of the derivative of the flow is dominated by the behavior of the flow in the linearized region.

3.3. The first return map to $S$. The above combined effects imply that the foliation of $S$ given by the lines $S \cap\left\{x=x_{0}\right\}$ is invariant under the Poincaré first return map $P: S^{*} \rightarrow S$, meaning that, for any given leaf $\gamma$ of this foliation, its image $P(\gamma)$ is contained in a leaf of the same foliation. Hence $P$ must have the form $P(x, y)=(f(x), g(x, y))$ for some functions $f: I \backslash\{0\} \rightarrow I$ and $g:(I \backslash\{0\}) \times I \rightarrow I$, where $I=[-1 / 2,1 / 2]$. 
Taking into account the definition of $L$ from the linearized region we see that

$$
f(x)=\left\{\begin{array}{ll}
f_{1}\left(x^{\alpha}\right), & \text { if } x<0 \\
f_{0}\left(x^{\alpha}\right), & \text { if } x>0
\end{array} ; \quad \text { with } f_{i}=(-1)^{i} a \cdot x+b_{i} \quad i=0,1 ;\right.
$$

for constants $a>0$ and $b_{0}, b_{1} \in(-1 / 2,1 / 2)$, and

$$
g(x, y)=\left\{\begin{array}{ll}
g_{1}\left(x^{\alpha}, y \cdot x^{\beta}\right), & \text { if } x<0 \\
g_{0}\left(x^{\alpha}, y \cdot x^{\beta}\right), & \text { if } x>0
\end{array},\right.
$$

where $g_{1} \mid I^{-} \times I \rightarrow I$ and $g_{0} \mid I^{+} \times I \rightarrow I$ are suitable affine maps, with $I^{-}=[-1 / 2,0)$, $I^{+}=(0,1 / 2]$.

3.4. Properties of the one-dimensional map $f$. Now we specify the properties of the one-dimensional map $f$ that follow from the previous construction. On the one hand

(f1) to imitate the symmetry of the Lorenz equations we take $f(-x)=-f(x)$. This is not essential in what follows and is not preserved under perturbation of the flow;

(f2) $f$ is discontinuous at $x=0$ with lateral limits $f\left(0^{-}\right)=+\frac{1}{2}$ and $f\left(0^{+}\right)=-\frac{1}{2}$ Hence

$$
f(0+)=b_{1}=-\frac{1}{2}, \quad f(0-)=b_{0}=\frac{1}{2} \quad \text { and } \quad f\left(\frac{1}{2}\right)=\frac{a}{2^{\alpha}}+b_{1} \leq \frac{1}{2},
$$

thus $0<a \leq 2^{\alpha}$. Since $D f(x)=a \alpha|x|^{\alpha-1}$, its minimum is $D F(1 / 2)=a \alpha 2^{1-\alpha}$ and to get $D f>1$ we must have $a \alpha 2^{1-\alpha}>1$.

(f3) The map $f$ is differentiable on $I \backslash\{0\}$ and $D f(x)>\sqrt{2}$;

to get this all we need is to choose

$$
\frac{2^{\alpha-1 / 2}}{\alpha}<a<2^{\alpha} \quad \text { so that } 2^{1 / 2} \alpha>1 \quad \text { or } \quad \alpha>1 / \sqrt{2}
$$

This imposes a restriction on $\alpha=-\lambda_{3} / \lambda_{1}$, thus the eigenvalue $\lambda_{3}$ cannot be too small with respect to the eigenvalue $\lambda_{1}$ at the singularity.

(f4) the lateral limits of $D f$ at $x=0$ are $D f\left(0^{-}\right)=+\infty$ and $D f\left(0^{+}\right)=-\infty$.

On the other hand $g: S^{*} \rightarrow I$ is defined in such a way that it contracts the second coordinate: $g_{y}^{\prime}(w) \leq \mu<1$ for all $w \in S^{*}$, and the rate of contraction of $g$ on the second coordinate should be much higher than the expansion rate of $f$. In addition the expansion rate is big enough to obtain a strong mixing property for $f$.

Remark 3.3. The expression of $D f$ ensures that the map $f$ satisfies

$$
|\log D f(x)-\log D f(y)|=(1-\alpha) \log \left|\frac{y}{x}\right|=(1-\alpha) \log \left|\frac{y-x}{y}+1\right| \leq \frac{1-\alpha}{|x|}|y-x|
$$

and also that $D f(x)=a \alpha|x|^{\alpha-1}$ which shows that $f$ behaves like a power of the distance to the singular set $\{0\}$. 
3.5. Properties of the map $g$. We note that by its definition the map $g$ is piecewise $C^{2}$ and we can obtain the following bounds on its partial derivatives:

(1) For all $(x, y) \in S^{*}$ with $x \neq 0$, we have $\left|\partial_{y} g(x, y)\right|=|x|^{\beta}$. As $\beta>1$ and $|x| \leq 1 / 2$ there is $0<\lambda<1$ such that

$$
\left|\partial_{y} g\right|<\lambda
$$

(2) For $(x, y) \in S^{*}$ with $x \neq 0$, we have $\partial_{x} g(x, y)=\beta x^{\beta-\alpha}$. Since $\beta>\alpha$ and $|x| \leq 1 / 2$ we get $\left|\partial_{x} g\right|<\infty$.

We note that from the first item above we have, first, a very strong domination of the contraction along the $y$-direction over the expansion along the $x$-direction, that is

$$
\frac{\left|\partial_{y} g(x, y)\right|}{|D f(x)|} \approx|x|^{\beta-\alpha+1} \approx \frac{|x|^{\beta}}{|D f(x)|} \text { with } \beta-\alpha+1>1 \text {. }
$$

Secondly, from this there follows the uniform contraction of the foliation $\mathcal{F}_{X}$ given by the lines $S \cap\{x=$ constant $\}$, that is: there exists a constant $C>0$ such that, for any given leaf $\gamma$ of the foliation and for $y_{1}, y_{2} \in \gamma$, then

$$
\operatorname{dist}\left(P^{n}\left(y_{1}\right), P^{n}\left(y_{2}\right)\right) \leq C \lambda^{n} \operatorname{dist}\left(y_{1}, y_{2}\right) \quad \text { when } n \rightarrow \infty .
$$

Thus the study of the maximal invariant set $\Lambda$ inside the trapping region

$$
U:=\left\{X^{t}(x, y, 1):(x, y, 1) \in S, 0 \leq t \leq \tau_{X}(x, y)\right\} \cup\{(0,0,0)\}
$$

for this 3 -flow can be reduced to the study of a bi-dimensional map, where $\tau_{X}$ is the first return time of the orbit of $(x, y, 1) \in S$ under $X^{t}$ to $S$. Moreover, the dynamics of this map can be further reduced to a one-dimensional map, because the invariant contracting foliation $\mathcal{F}_{X}$ enables us to identify two points on the same leaf, since their orbits remain forever on the same leaf and the distance of their images tends to zero under iteration. See Figure 3 for a sketch of this identification.

The quotient map $f: S^{*} / \mathcal{F}_{X} \rightarrow S / \mathcal{F}_{X}$ obtained through the identification $\pi: S \rightarrow S / \mathcal{F}_{X}$ will be called the (one-dimensional) Lorenz map. It satisfies $f \circ \pi=\pi \circ P$ by construction and we note that $S^{*} / \mathcal{F}_{X}$ is naturally identified with $I \backslash\{0\}$ through a diffeomorphism, so that we obtain in fact the first component of the Poincaré return map $P$ to $S$. Figure 3 shows the graph of this one-dimensional transformation.

3.6. Persistence and smoothness of the contracting foliation. The following persistence property is a consequence of the domination of the contraction along the $y$-direction over the expansion along the $x$-direction (see e.g. [7]).

Theorem 3.4. Let $X$ be the vector field obtained in the construction of the geometric Lorenz model and $\mathcal{F}_{X}$ the invariant contracting foliation of the cross-section $S$. Then any vector field $Y$ which is sufficiently $C^{1}$-close to $X$ admits an invariant contracting continuous foliation $\mathcal{F}_{Y}$ on the cross-section $S$ with $C^{1}$ leaves.

It can be shown that the holonomies along the leaves are in fact Hölder- $C^{1}$ (see e.g [7]). Moreover, under a strong dissipative condition on the eigenvalues of the equilibrium $\sigma$, that is, $\beta>\alpha+k$ for some $k \in \mathbb{Z}^{+}$(recall equation (4)) it can be proved, following [26] 

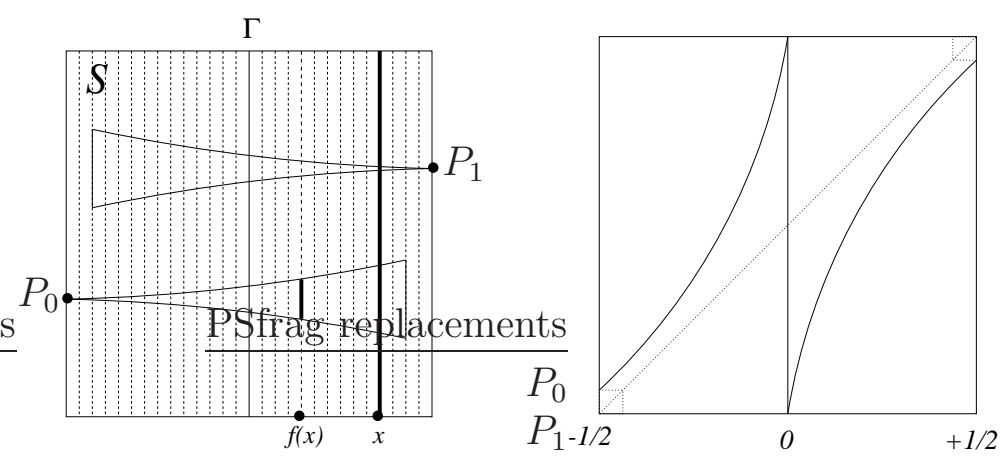

Figure 3. On the left: projection on $I$ through the stable leaves and a sketch of the image of one leaf under the return map. On the right: the Lorenz map $f$.

and [42], that $\mathcal{F}_{Y}$ is then a $C^{k}$ smooth foliation and so the holonomies along its leaves are $C^{k}$ maps. This is just an application of $k$-normal hyperbolicity to flows. We deduce the following.

Theorem 3.5. For strongly dissipative Lorenz attractors, with $\beta>\alpha+k$, the onedimensional quotient map $f$ is $C^{k}$ smooth away from the singularity. Moreover, this smoothness property is also persistent for all nearby $C^{k}$ flows, since the condition $\beta>\alpha+k$ is open in the $C^{1}$ topology.

In what follows we fix $k \geq 2$, which guarantees that the foliation $\mathcal{F}_{Y}$ is $C^{2}$ for all $Y$ in a $C^{2}$ neighborhood $\mathcal{U}$ of $X$. Moreover the one-dimensional piecewise expanding map $f_{Y}$ given as the quotient map of the corresponding Poincaré map $P_{Y}$ over the leaves of the foliation $\mathcal{F}_{Y}$ associated to $Y \in \mathcal{U}$.

3.7. Robust transitivity properties. Here we show that the Lorenz flows constructed are suspension flows with a roof function that is constant along the stable foliation on the cross-section $S$. First we discuss the case of the geometric Lorenz flow $X^{t}$ constructed in the previous section and then complete the proof of Proposition 3.1.

First, we observe that $S$ is, for the geometric Lorenz flow $X^{t}$ constructed in the previous sections, a collection of strong-stable leafs of the flow. Indeed, we can write

$$
S=\bigcup_{-1 / 2 \leq x \leq 1 / 2} W_{X, 1 / 2}^{s s}(x, 0,1)
$$

as a family of local strong-stable leaves for the vector field $X$ with radius $1 / 2$ through the points in $I \times\{0\} \times\{1\}$, the orthogonal segment to $\Gamma$ in $S$; see the left hand side of Figure 3 , Since the strong-stable foliation $\mathcal{F}_{Y}$ for $C^{2}$ close vector fields $Y$ is also a $C^{2}$ foliation, then we can repeat the construction with respect to every close vector field $Y$ and obtain a smooth surface

$$
S_{Y}:=\bigcup_{-1 / 2 \leq x \leq 1 / 2} W_{Y, 1 / 2+\varepsilon}^{s s}(x, 0,1)
$$


which is a cross-section for the flow $Y^{t}$, and contains the continuation of the points $P_{1}, P_{0}$ as the first visits of the branches of the unstable manifold $W_{Y}^{u}\left(\sigma_{Y}\right)$ of the singularity to the cross-section, for small enough $\varepsilon>0$; see Figure 3 .

Moreover we can, by the $C^{2}$ change of coordinates which linearizes the flow around $\sigma_{Y}$, assume first that the new singularity $\sigma_{Y}$ is still at the origin, and that on $S_{Y}$ we have coordinates $(x, y)$ such that $x=$ const represents a curve on $S_{Y}$ which is uniformly contracted by the Poincaré return map associated to $Y$; in fact, $x=$ const is precisely the strong-stable manifold through $(x, 0,1)$. In particular, this ensures that the Poincaré return time of points on $S_{Y}$ to $S_{Y}$ in the same leaf of $\mathcal{F}_{Y}$ is constant, for all $Y$ sufficiently $C^{2}$ close to $X$. This extends a very useful property of $X$ to all nearby vector fields.

In what follows $|x|$ still represents the distance of the curve $x=$ const to $\Gamma_{Y}:=S_{Y} \cap$ $W_{l o c}^{s}\left(\sigma_{Y}\right)$, the intersection of the local stable manifold of the singularity with $S_{Y}$. The Poincaré return time of a point $\left(x_{0}, y_{0}\right)$ in $S_{Y}^{*}:=S_{Y} \backslash \Gamma_{Y}$ equals

$$
\tau_{Y}\left(x_{0}, y_{0}\right)=-\frac{1}{\lambda_{1}(Y)} \log \left|x_{0}\right|+s_{Y}\left(x_{0}\right),
$$

where $s_{Y}: S_{Y}^{*} \rightarrow \mathbb{R}$ is $C^{2}$ close to $s_{X}=$ const.

Let $f_{Y}$ be the one-dimensional map as the quotient map of the corresponding Poincaré map $P_{Y}$ over the leaves of the foliation $\mathcal{F}_{Y}$ for all flows $Y$ close to $X$ in the $C^{1}$ topology as above. Since the leaves of $\mathcal{F}_{Y}$ are $C^{1}$ close to those of $\mathcal{F}$, it follows that $f_{Y}$ is $C^{1}$ close to $f$ and thus there exists $c \in[-1 / 2,1 / 2]$ which plays for $f_{Y}$ the same role of the singular point at 0 , so that, after a linear change of coordinates, we can assume that $c=0$ and properties (f2)-(f4) from Subsection 3.4 are still valid, albeit with different constants, for $f_{Y}$ on a subinterval $\left[-b_{0}, b_{1}\right]$ for some $0<b_{0}, b_{1}<1 / 2$ close to $1 / 2$. In particular

$$
D f_{Y}(x) \approx|x|^{\alpha-1} \quad \text { i.e. } \quad \frac{1}{C} \leq \frac{D f_{Y}(x)}{|x|^{\alpha-1}} \leq C
$$

for some $C>1$ uniformly on a $C^{2}$ neighborhood of $X$, where $\alpha=\alpha(Y)=-\lambda_{3}(Y) / \lambda_{1}(Y)$ depends smoothly on vector field. Finally, the condition (f3) ensures that $f_{Y}$ has enough expansion to easily prove that every $f_{Y}$ is locally eventually onto for all $Y$ close to $X$. More precisely,

Lemma 3.6. [7, Lemma 3.16] For any interval $J \subset\left(-b_{0}, b_{1}\right)$ there exists an iterate $n \geq 1$ such that $f_{Y}^{n}(J)=\left(0, b_{1}\right)$ or $f_{Y}^{n}(J)=\left(-b_{0}, 0\right)$; and the next iterate covers $\left(f\left(-b_{0}\right), f\left(b_{1}\right)\right)$.

In particular, this implies that $f_{Y}$ is transitive and, as well known, $\Lambda_{Y}$ turns out to be transitive also. So we have a robust transitive attractor on a $C^{2}$ neighborhood of $X$.

Remark 3.7. Lemma 3.6 implies also that every given point $q$ of $\left(-b_{0}, b_{1}\right)$ belongs to the some positive image $f^{n}(J)$ of any given interval $J \not \supset 0$, for some $n>0$. Hence every $q \in\left(-b_{0}, b_{1}\right)$ has a dense set of pre-images, that is $\overline{\cup_{k \geq 0} f_{Y}^{-k}(\{q\})}=\left[-b_{0}, b_{1}\right]$.

Taken together with the results in the previous subsections we proved Proposition 3.1 .

Remark 3.8. Given $\delta>0$ there exists an integer $N$ such that $O_{N}(Y):=\cup_{i=1}^{N}\left(f_{Y}^{i}\right)^{-1}(\{0\})$ is $2 \delta$-dense in $\left(-b_{0}, b_{1}\right)$. Moreover, by slightly modifying the return to $S$ from $\Sigma^{ \pm}$(see 
Section 3.2) during the construction of $X$, we can assume that the singular point 0 of $f_{Y}$ does not belong to $O_{N}(Y)$ and $O_{N}(Y)$ is $\delta$-dense in $I$, for each vector field $Y$ in a $C^{2}$ neighborhood $\mathcal{U}$ of the original geometric Lorenz flow $X$.

We can, by another smooth change of coordinates, assume without loss of generality that both $b_{0}, b_{1}$ equal $1 / 2$ in what follows.

\section{The GeOMetric LOREnZ Flow IS A GOOD HYPERBOliC SKEW-PRODUCT}

Now we explain step by step how to obtain all the required properties to conclude that the geometric Lorenz flows constructed in Section 3 are good hyperbolic skew-products.

We fix $Y$ a vector field in a $C^{2}$ neighborhood $\mathcal{U}$ of $X$, with a geometric Lorenz attractor $\Lambda$ in a trapping region $U$, which contains the image of the cross-section $S$ under the flow $Y^{t}$ until its points first return to $S$. We denote by $P_{Y}: S_{Y}^{*} \rightarrow S_{Y}$ the associated Poincaré first return map, and by $f_{Y}: I \backslash\{0\} \rightarrow I$, with $I=[-1 / 2,1 / 2]$, the corresponding Lorenz map obtained by the action of $P_{Y}$ on the leaves $\mathcal{F}_{Y}$ of the contracting foliation on $S^{*}=S_{Y}^{*}$. We also set $\tau=\tau_{Y}: S^{*} \rightarrow\left[\tau_{0},+\infty\right)$ the first return time function associated to $P=P_{Y}$ so that $P(w)=Y^{\tau(w)}(w), w \in S^{*}$.

4.1. The uniformly expanding Markov map. We show that a Lorenz map $f=f_{Y}$ admits an induced map $F$ on a small interval $\Delta \subset I$ which is a uniformly expanding Markov map. Induced Markov transformations for $C^{1+\alpha}$ Lorenz transformations were obtained first by [17] but that construction is not suitable for our estimates. Indeed, a crucial property, the uniform non-integrability, is obtained in Subsection 4.2.3 using essentially that $\operatorname{Dr}(x) \approx|x|^{-1}$ while $D f(x) \approx|x|^{\alpha-1}$ for $x$ near the zero, so their growth rates are significantly different near the singularity. To take advantage of this we build an induced map on an interval $\Delta$ which is an open neighborhood of the singular point at the origin. To the best of our knowledge this type of construction is not available in the literature, but can be obtained using a number of other results as follows.

We assume that $f$ satisfies the properties given in Section 3.4 and follow the exposition in [3, 23]. Let $b$ be a fixed constant satisfying $0<b<\min \{1 / 2,1 /(4|1-\alpha|)\}$.

We start by observing that $f$ is a local diffeomorphism away from 0 and behaves like a power of the distance to the singular set; see Remark 3.3. This says that 0 is a "nondegenerate singularity" according to [2], a concept generalized from one-dimensional dynamics.

Given $\xi, \sigma \in(0,1)$ and $\delta>0$, we say that $n$ is a $(\sigma, \delta)$-hyperbolic time for a point $x \in I$ if, for all $1 \leq k \leq n$

$$
\prod_{j=n-k}^{n-1}\left\|D f\left(f^{j}(x)\right)^{-1}\right\| \leq \sigma^{k} \quad \text { and } \quad\left|f^{n-k}(x)\right|_{\delta} \geq \sigma^{b k}
$$

where $|z|_{\delta}=|z|$ if $|z|<\delta$ and $|z|_{\delta}=1$ otherwise.

We present well-established results showing that (i) if $n$ is a hyperbolic time for $x$, the map $f^{n}$ is a diffeomorphism with uniformly bounded distortion on a neighborhood of $x$ that is mapped to a disk of uniform radius; (ii) Lebesgue almost every point has many 
hyperbolic times for the one-dimensional Lorenz transformation $f$. We say that $f^{n}$ has bounded distortion by a factor $D$ on a set $V$ if, for every $x, y \in V$,

$$
\frac{1}{D} \leq \frac{\left|D f^{n}(x)\right|}{\left|D f^{n}(y)\right|} \leq D
$$

Lemma 4.1. Given $\sigma \in(0,1)$ and $\delta>0$, there exist $\delta_{1}, D_{1}, \kappa>0$ (depending only on $\sigma, \delta$ and on the map $f$ ) such that for any $x \in M$ and $n \geq 1$ a $(\sigma, \delta)$-hyperbolic time for $x$, there exists a neighborhood $V_{n}(x)$ of $x$ with the following properties:

(1) $f^{n}$ maps $V_{n}(x)$ diffeomorphically onto the ball $B\left(f^{n}(x), \delta_{1}\right)$;

(2) for $1 \leq k<n$ and $y, z \in V_{n}(x),\left|f^{n-k}(y)-f^{n-k}(z)\right| \leq \sigma^{k}\left|f^{n}(y)-f^{n}(z)\right|$;

(3) $f^{n}$ has distortion bounded by $D_{1}$ on $V_{n}(x)$;

(4) $V_{n}(x) \subset B\left(x, 2 \delta_{1} \sigma^{n}\right)$.

Proof. For the proofs of items 1, 2, 3 see Lemma 5.2 and Corollary 5.3 in [2]. Item 4 is an immediate consequence of the backward contraction property at item 2 .

We say that the sets $V_{n}(x)$ are hyperbolic pre-balls and their images $f^{n}\left(V_{n}(x)\right)$ are hyperbolic balls; the latter are indeed balls of radius $\delta_{1}$. For the existence of hyperbolic times, we observe that

- $f$ is a $C^{2}$ piecewise expanding map since $|D f|>\sqrt{2}>1$;

- It follows from [17] that $C^{2}$ Lorenz transformations have an unique absolutely continuous invariant measure $\nu_{0}$ with Lyapunov exponent $\lambda\left(\nu_{0}\right)=\int \log \left|f^{\prime}\right| d \nu_{0}>0$ whose basin $B\left(\nu_{0}\right)$ covers Lebesgue almost every point;

- Since $|D f(x)| \approx|x|^{\alpha-1}$ behaves like a power of the distance to the singular point then $\log |x|$ is $\nu_{0}$-integrable and, by the Ergodic Theorem, for every $\varepsilon>0$ we can find $\delta>0$ such that

$$
\lim _{n \rightarrow+\infty} \frac{1}{n} \sum_{i=0}^{n-1}-\log \left|f^{i}(x)\right|_{\delta}=\int_{-\delta}^{\delta}-\log |x| d \nu_{0}(x)<\varepsilon
$$

for every $x \in B(\mu)$, thus for Lebesgue almost every $x$.

Condition (11) is known as a slow recurrence condition on the singular set $\{0\}$ of $f$. Under these conditions, together with the non-degeneracy property given by Remark 3.3, we have

Lemma 4.2. There exists $\theta>0$ and $0<\delta<1$ (depending only on $f$ and the expanding rate $\sqrt{2}$ ) such that, for Lebesgue almost every $x \in I$, we can find $n_{0} \geq 1$ satisfying: for each $n>n_{0}$ there are $(\sigma, \delta)$-hyperbolic times $1 \leq n_{1}<\cdots<n_{l} \leq n$ for $x$ with $l \geq \theta n$.

Proof. See Lemma 5.4 of [2]. Let us remark that here we have $\sigma=1 / \sqrt{2} \approx 0.707$.

Notice that the constants $\alpha, \beta$ and the lower bound for the expansion rate $\sqrt{2}$ vary slightly in a $C^{2}$ neighborhood $\mathcal{U}$ of the geometric Lorenz flow $X$. Likewise, the value of $\delta_{1}$ from Lemma 4.1 depends continuously $\alpha, \beta$ and inf $|D f|$, and so we can assume that $\delta_{1}=\delta_{1}(Y)>\underline{\delta}_{1}$ for some uniform constant $\underline{\delta}_{1}>0$ for all $Y \in \mathcal{U}$.

Hence, from Remark 3.8 and Section 3.6, we obtain a neighborhood $\mathcal{U}$ of the geometric Lorenz flow $X$ in $\mathfrak{X}^{2}(M)$ such that, for all $Y \in \mathcal{U}$, the set $O_{N}(Y)$ of $N$-pre-images of $\{0\}$ 
under $f_{Y}$ is $\underline{\delta}_{1} / 3$-dense in $I$ and does not contain the singular point. Thus the point 0 and the map $f$ satisfy all the conditions needed to perform the construction of an induced uniformly expanding Markov map $F$ from a neighborhood $\Delta=(-a, a)$ of 0 to itself, as presented in [3, 23]. More precisely,

Theorem 4.3. There exists a neighborhood $\Delta:=(-a, a)$, for some $0<a<1 / 2$, of the singular point 0 ; a countable Lebesgue modulo zero partition $\mathcal{Q}$ of $\Delta$ into sub-intervals; $a$ function $R: \Delta \rightarrow \mathbb{Z}^{+}$defined almost everywhere, constant on elements of the partition $\mathcal{Q}$; and constants $c>0, \kappa>1$ such that, for all $\omega \in \mathcal{Q}$ and $R=R(\omega)$, the map $F:=f^{R}$ : $\omega \rightarrow \Delta$ is a $C^{2}$ diffeomorphism, satisfies the bounded distortion property and is uniformly expanding: for each $x, y \in \omega$

$$
\left|\frac{D f^{R}(x)}{D f^{R}(y)}-1\right| \leq c\left|f^{R}(x)-f^{R}(y)\right| \quad \text { and } \quad\left|f^{R}(x)-f^{R}(y)\right|>\kappa|x-y| .
$$

Moreover, for each $\omega \in \mathcal{Q}$ there exists $0<k \leq N$ such that $n:=R(\omega)-k$ is a $\left(\sigma, \delta_{1}\right)$ hyperbolic time for each $x \in \omega$; $\omega \subset V_{n}(x)$ and, in addition, $f^{j}(\omega) \subset I \backslash \Delta$ for all $n \leq j<R(\omega)$.

It was proved in [4] that the one-dimensional Lorenz transformation has exponentially slow recurrence to the singular set, that is, for every $\varepsilon>0$ there exists $\delta>0$ such that

$$
\left.\limsup _{n \rightarrow+\infty} \frac{1}{n} \log \lambda\left(\left.\left\{x \in I: \frac{1}{n} \sum_{i=0}^{n-1}-\log \mid f^{i}(x)\right)\right|_{\delta}>\varepsilon\right\}\right)<0 .
$$

Following [23] this ensures the following result.

Theorem 4.4. In the same setting of the previous Theorem 4.3, the inducing time function $R$ has exponential tail, that is, there exists positive constants $c$ and $\gamma$ such that

$$
\lambda(\{x \in I: R(x)>n\})<c e^{-\gamma n} .
$$

This Markov map $F$ is obtained by inducing the interval map $f$ on the interval $\Delta$, using an inducing time that is given by the sum of a hyperbolic time with a non-negative integer bounded by the number $N$ defined in (3.8), and has exponential tail with respect to the Lebesgue measure. Therefore, $R$ is Lebesgue integrable and the following is well-known.

Proposition 4.5. There exists an absolutely continuous invariant probability measure $\nu$ for $F$ whose density $\phi=d \nu / d \lambda$ is a $C^{1}$ strictly positive and bounded function on $\Delta$. Moreover, $\nu_{0}=\frac{1}{\int R d \nu} \cdot \sum_{k=0}^{\infty}\left(f^{k}\right)_{*}(\nu \mid R>k)$.

4.1.1. Renyi condition and $C^{1}$ invariant density. The previous induced map $F$ satisfies a stronger property than the expression above for bounded distortion, the Renyi condition from [40]. Indeed, by a simple computation

$$
\frac{\left|D^{2} F\right|}{|D F|^{2}}(x) \leq \sum_{j=0}^{R(x)-1} \frac{1}{|D F(x)|} \frac{\mid D^{2} f\left(f^{j}(x) \mid\right.}{\mid D f\left(f^{j}(x) \mid\right.}
$$


Therefore we can obtain the uniform upper bound

$$
\begin{aligned}
\frac{\left|D^{2} F\right|}{|D F|^{2}}(x) & \leq \sum_{i=0}^{R-1} \frac{1}{\left|D f^{R-i}\left(f^{i}(x)\right)\right|} \cdot \frac{\left|D^{2} f\left(f^{i}(x)\right)\right|}{\left|D f\left(f^{i}(x)\right)\right|^{2}} \lesssim \sum_{i=0}^{R-1} \sigma^{R-i} \frac{\left|f^{i}(x)\right|^{\alpha-2}}{\left(\left|f^{i}(x)\right|^{\alpha-1}\right)^{2}} \\
& =\sum_{i=0}^{R-1} \frac{\sigma^{R-i}}{\left|f^{i}(x)\right|^{\alpha}} \leq \sum_{i=0}^{R-1} \frac{\sigma^{R-i}}{\sigma^{\alpha b(R-i)}} \leq B=\frac{1-\alpha}{a \alpha} \sum_{i \geq 0} \sigma^{(1-b \alpha) i}<\infty
\end{aligned}
$$

for every $x \in \omega$ and $\omega \in \mathcal{Q}$, where $R=R(\omega)$. This implies that, for $x, y \in \omega$

$$
\left|\frac{1}{D F}(x)-\frac{1}{D F}(y)\right| \leq|x-y| \frac{\left|D^{2} F\right|}{|D F|^{2}}(z) \leq B|x-y|
$$

for some $z \in \omega$ given by the Mean Value Theorem. Moreover, given $n>1$

$$
\begin{aligned}
\left|\frac{D^{2} F^{n}(x)}{\left(D F^{n}(x)\right)^{2}}\right| & =\frac{1}{\left|D F^{n}(x)\right|^{2}}\left|\sum_{i=0}^{n-1} D^{2} F\left(F^{i}(x)\right)\left(\prod_{\substack{j=0, \ldots, n-1 \\
j \neq i}} D F\left(F^{j}(x)\right)\right)\right| \\
& =\frac{1}{\left|D F^{n}(x)\right|} \sum_{i=0}^{n-1} \frac{\left|D^{2} F\left(F^{i}(x)\right)\right|}{\left|D F\left(F^{i}(x)\right)\right|} \leq \frac{B}{\left|D F^{n}(x)\right|} \sum_{i=0}^{n-1}\left|D F\left(F^{i}(x)\right)\right| \\
& =B \sum_{i=0}^{n-1} \frac{\left|D F\left(F^{i}(x)\right)\right|}{\left|D F^{n}(x)\right|} \leq B \cdot n \cdot \sigma^{n-1}
\end{aligned}
$$

which is an infinitesimal when $n \rightarrow+\infty$.

So, we get that there exists a $C^{2}$ uniformly expanding Markov map $F$ satisfying the Renyi condition from the previous remark. In this setting the arguments of Baladi-Vallée from [10] provide an invariant density for $F$ in the space of $C^{1}$ functions.

Lemma 4.6. The density $\phi=d \nu / d \lambda$ of the F-invariant probability measure $\nu$ is a $C^{1}$ function $\phi:[-1 / 2,1 / 2] \rightarrow[0,+\infty)$.

4.1.2. Uniform bounded distortion for powers of the induced map. We need this technical result in the final arguments and we are ready to prove it here.

Proposition 4.7. There exists $B_{0}>0$ such that for all $n \in \mathbb{Z}^{+}, h \in \mathcal{H}_{n}$ and $x, y \in I$

$$
\left|\frac{D F^{n}(h(x))}{D F^{n}(h(y))}\right| \leq B_{0}|x-y| .
$$

Proof. We use the properties of hyperbolic times and the last part of the statement of Theorem 4.3 to explicitly estimate this bound. First, we fix $n \in \mathbb{Z}^{+}$and set $R_{i}:=R\left(\omega_{i}\right)$ where $\omega_{i}=\mathcal{Q}\left(F^{i}(h(y))\right)$ is the element of the partition $\mathcal{Q}$ containing both $F^{i}(h(y)), F^{i}(h(x))$, $i=0, \ldots, n-1$. Secondly, we set $n_{i}:=R_{i}-k_{i}$ to be the hyperbolic time of $F^{i}(h(x))$ given 
by Theorem 4.3. Then we write $\bar{x}:=h(x), \bar{y}=h(y)$ and

$$
\log \left|\frac{D F^{n}(\bar{x})}{D F^{n}(\bar{y})}\right|=\sum_{i=0}^{n-1} \sum_{j=0}^{R_{i}-1} \log \left|\frac{D f\left(f^{j}\left(F^{i}(\bar{x})\right)\right)}{D f\left(f^{j}\left(F^{i}(\bar{y})\right)\right)}\right|
$$

where on hyperbolic times the summand can be bounded as follows

$$
\begin{aligned}
\log \left|\frac{D f\left(f^{j}\left(F^{i}(\bar{x})\right)\right)}{D f\left(f^{j}\left(F^{i}(\bar{y})\right)\right)}\right| & \approx \log \left|\frac{f^{j}\left(F^{i}(\bar{x})\right)}{f^{j}\left(F^{i}(\bar{y})\right)}\right| \leq \frac{\left|f^{j}\left(F^{i}(\bar{x})\right)-f^{j}\left(F^{i}(\bar{y})\right)\right|}{\left|f^{j}\left(F^{i}(\bar{y})\right)\right|} \\
& \leq \frac{\sigma^{n_{i}-j}\left|f^{n_{i}}\left(F^{i}(\bar{x})\right)-f^{n_{i}}\left(F^{i}(\bar{y})\right)\right|}{\sigma^{b\left(n_{i}-j\right)}} \leq \sigma^{b\left(R_{i}-j\right)}\left|F^{i+1}(\bar{x})-F^{i+1}(\bar{y})\right|
\end{aligned}
$$

since $f$ expands distances by at least $\sigma^{-1}$ on both branches. Putting this inequality in the summation above we get

$$
\log \left|\frac{D F^{n}(\bar{x})}{D F^{n}(\bar{y})}\right| \leq \frac{\sigma^{b}}{1-\sigma^{b}} \sum_{i=0}^{n-1}\left|F^{i+1}(\bar{x})-F^{i+1}(\bar{y})\right| .
$$

But $F$ also expands distances inside each partition element, thus

$$
\log \left|\frac{D F^{n}(\bar{x})}{D F^{n}(\bar{y})}\right| \leq \frac{\sigma^{b}}{1-\sigma^{b}}|x-y| \sum_{i=0}^{n-1} \kappa^{-(n-i)} \leq \frac{\sigma^{b}}{1-\sigma^{b}} \frac{\kappa^{-1}}{1-\kappa^{-1}}|x-y| .
$$

To complete the proof it is enough to define $B_{0}:=\sigma^{b}\left(1-\sigma^{b}\right)^{-1}(\kappa-1)^{-1}$.

4.2. The good roof function. Here we show that the associated flow return time function $r: \cup_{\omega \in \mathcal{Q}} \omega \rightarrow\left(r_{0},+\infty\right)$, where $r_{0}>0$ depends only on $f$, induced from $\tau$ and associated to the induced map $F$, is a good roof function. Note that the function $r$ is defined from the Poincaré return time function for every $x \in \bigcup_{\omega \in \mathcal{Q}} \omega$ as

$$
r(x)=S_{R} \varrho(x):=\sum_{j=0}^{R(x)-1} \varrho\left(f^{j}(x)\right)
$$

where $\varrho(x):=\inf \left\{\tau(z): z \in \pi^{-1}(\{x\})\right\}=\tau(x, 0,1)$, for $x \in I \backslash\{0\}$, since $\tau$ does not depend of the point we choose on some strong-stable leaf in $S^{*}$. Clearly $r$ is still bounded from below by the same value $\tau_{0}$ that bounds $\tau$ on each linearized flow box near the origin; see Section 3.1. This is property (1) of a good roof function.

Furthermore, as a consequence of our construction in Section 3 and expressed in (9), there exists a function $s_{Y}$, constant on strong-stable leafs, which is $C^{2}$ close to a constant function on $S_{Y}^{*}$ such that for every $Y C^{2}$-close to

$$
\varrho_{Y}(x)=-\frac{1}{\lambda_{1}(Y)} \log |x|+s_{Y}(x) .
$$

As a consequence we obtain that

$$
\left|D \varrho_{Y}(x)+\left(\lambda_{1}(Y) x\right)^{-1}\right|=\left|D s_{Y}(x)\right|, x \in I \backslash\{0\}
$$


is uniformly $C^{1}$ close to the zero function. In particular, we can find $\xi>0$ so that $|D s(x)| \leq \xi|x|^{-1}$ and hence there are $\xi_{1}, \xi_{2}>0$ such that

$$
\xi_{1} \leq-x \cdot D \varrho(x) \leq \xi_{2},
$$

Notice also that one can take $\left|\xi_{i}-1 / \lambda_{1}(X)\right|$ as small as needed, for $i=1,2$, by taking $Y$ sufficiently $C^{2}$ close to $X$.

4.2.1. Exponential tail. We split the estimates in three cases depending on how large the tail constant is. Consider the positive real $\xi=(2 \nu(\rho))^{-1}=\left(2 \int \rho d \nu\right)^{-1}$ and take a positive integer $L$.

Case $R$ big enough: If $R>\xi L$, then

$$
\operatorname{Leb}\{r>L \& R>\xi L\} \leq \operatorname{Leb}\{R>\xi L\}=\operatorname{Leb}\left(\bigcup_{\omega \in \mathcal{P}, R(\omega)>\xi L} \omega\right) \leq c e^{-\gamma \xi L}
$$

since $R$ has exponential tail.

Case $R$ not so big: If $R \leq \xi L$, then $S_{R} \varrho>L$ implies that $S_{R} \varrho-\nu(\varrho)>L-\nu(\varrho)$ and

$$
\frac{1}{R} \sum_{i=0}^{R-1}\left(\varrho \circ f^{i}-\nu(\varrho)\right)>\frac{L-\nu(\varrho)}{R}>\frac{L}{R}-\nu(\varrho)>\frac{1}{\xi}-\nu(\varrho)=\nu(\varrho)>0
$$

and also $\nu\left(\varrho \circ f^{i}-\nu(\varrho)\right)=\int\left(\varrho \circ f^{i}-\int \varrho d \nu\right) d \nu=0$.

At this point we recall a large deviations result for non-uniformly expanding maps (see e.g. [6, 4, 47]) which guarantees that

$$
\limsup _{n \rightarrow+\infty} \frac{1}{n} \log \operatorname{Leb}\left\{x: \frac{1}{n} \sum_{i=0}^{n-1} \varrho \circ f^{i}(x)>2 \nu(\varrho)>0\right\}<0
$$

so that the measure $\operatorname{Leb}\left\{x: \frac{1}{R} \sum_{i=0}^{R-1} \varrho \circ f^{i}(x)>2 \nu(\varrho)\right\}$ is exponentially small in $R$ (thus in $\xi L$ also), but only for $R \geq R_{0}$, for some integer $R_{0}$. We remark that $R_{0}$ does not depend on the value of $L$. Thus we have achieved an exponential tail for

$$
\operatorname{Leb}\left\{x \in \Delta: R_{0} \leq R(x) \leq \xi L \& r>L\right\} .
$$

Case $R$ small $\left(R<R_{0}\right)$ : It is enough to consider the case $L \gg R_{0}$ since we are only interested on the measure of the tail set of $r$. Hence $r>L \Longleftrightarrow \sum_{i=0}^{R-1} \varrho \circ f^{i}>L$ implies $\varrho \circ f^{i}>L / R_{0}$ for some $i \in\left\{0, \ldots, R_{0}\right\}$. Hence

$$
\operatorname{Leb}\left\{r>L \& R \leq R_{0}\right\} \leq \operatorname{Leb}\left\{\varrho \circ f^{i}>L / R_{0}\right\}=\left(f_{*}^{i} \operatorname{Leb}\right)\left\{\varrho>L / R_{0}\right\} .
$$

Since $|D f|>\sqrt{2}, f$ has two $C^{2}$ monotonous branches and $0 \leq i \leq R_{0}$, we have that the density of $f_{*}^{i}$ Leb is smaller than $2^{i / 2}$ : for each branch $f \mid I^{ \pm}$we have $\left(f \mid I^{ \pm}\right)_{*}$ Leb with density smaller than $2^{-1 / 2} ; f$ is not Markov and $f^{i}$ has $2^{i}$ branches whose images might intersect, so that the maximum density would be, in the worst case 
where the image of every branch intersects at some region, smaller than $2^{i} \cdot 2^{-i / 2}=$ $2^{i / 2}$. We conclude

$$
\begin{aligned}
\operatorname{Leb}\left\{r>L \& R \leq R_{0}\right\} & \leq 2^{i / 2} \operatorname{Leb}\left\{\varrho>L / R_{0}\right) \\
& \leq 2^{R_{0}} \operatorname{Leb}\left\{\varrho>L / R_{0}\right\} \leq 2^{R_{0}} \cdot e^{-\lambda_{1} L / R_{0}} .
\end{aligned}
$$

At this point we use (14) and conclude that the measure $\operatorname{Leb}\left\{r>L \& R \leq R_{0}\right\}$ decays exponentially fast with $L$.

This estimate together with (16) and (17) shows that $r$ has exponential tail.

4.2.2. The uniform bound of the derivative. For property (2) of a good roof function, let $h \in \mathcal{H}_{1}, h: \Delta \rightarrow \omega$ be an inverse branch of $F=f^{R}$ with inducing time $l=R(\omega) \geq 1$ and let us fix $x \in \omega$. Then

$$
|D(r \circ h)(x)|=|D r(h(x))| \cdot|D h(x)|=\frac{|D r(h(x))|}{|D F(h(x))|}=\left|\sum_{i=0}^{l-1} \frac{\left(D \varrho \circ f^{i}\right) \cdot D f^{i}}{D F} \circ h(x)\right| \text {. }
$$

In addition, from the construction of the inducing partition using hyperbolic times, we have $l=(l-n)+n$, where $l-n$ is a $\left(\sigma, \delta_{1}\right)$-hyperbolic time for $x_{0}$ and $0<n \leq N$. Thus $\left|x_{i}\right| \geq \sigma^{b(l-i)}$ for $x_{i}=f^{i}(h(x))$ by definition of hyperbolic times, and so, by (15) we get $\left|D \varrho\left(x_{i}\right)\right| \leq \xi_{2} \sigma^{-b(l-i)}, i=0, \ldots, l-n$, where $\sigma=1 / \sqrt{2}$. Moreover

$$
\left|\frac{D f^{i}}{D F}\right| \circ h(x)=\frac{1}{\left|D f^{l-i} \circ f^{i}\right|} \circ h(x) \leq \sigma^{(l-i)}, \quad i=0, \ldots, l-1 ;
$$

and $\left|x_{i}\right| \geq \delta$ and $D f\left(x_{i}\right)>\sigma^{-1}$ for $l-n<i<R(\omega)$. Altogether this implies, because $0<b<1 / 2$, that $|D(r \circ h)(x)| \leq\left(\xi_{2} / \delta\right) \sum_{i=0}^{l-1} \sigma^{(1-b) i}$ which is bounded by a constant. Thus we have proved that $\sup _{h \in \mathcal{H}_{1}}\|D(r \circ h)\|_{0}$ is finite.

4.2.3. Uniform non-integrability. We prove that $r$ satisfies the aperiodicity condition, the third item in Definition 2.1, for a $C^{k}$ open set of vector fields, $k \geq 2$, throught a perturbative argument.

If there exists a $C^{1}$ function $u: \Delta \rightarrow \mathbb{R}$ and a measurable function $v: \Delta \rightarrow \mathbb{R}$ constant on each element $\omega$ of $\mathcal{Q}$ satisfying $r=u \circ F-u+v$, then we show that, up to a $C^{1}$ perturbation of the original vector field $X$, this is impossible for all $C^{2}$ nearby vector fields. For this we choose two distinct periodic points $x_{1}, x_{2}$ for $F: \Delta \circlearrowleft$ of the same period $n$ whose orbits are (i) distinct, and (ii) each orbit visits each of the elements of the Markov partition the same number of times as the other, but (iii) necessarily in some different order to each other. The existence of such a pair of periodic orbits is a consequence of $F$ being a full branch Markov map: if $\omega_{1}, \omega_{2}$ are two elements of the Markov partition, we can choose the period $p=4$ and $x_{i}, i=1,2$ such that

$$
x_{1}, F\left(x_{1}\right) \in \omega_{1}, F^{2}\left(x_{1}\right), F^{3}\left(x_{1}\right) \in \omega_{2} \quad \text { and } \quad x_{2}, F^{2}\left(x_{2}\right) \in \omega_{1}, F\left(x_{2}\right), F^{3}\left(x_{2}\right) \in \omega_{2} .
$$

Furthermore, $x_{1}, x_{2}$ can be chosen in the interior of $\omega_{1}$. The cohomological equation implies $S_{p} r\left(x_{1}\right)=S_{p} v\left(x_{1}\right)=S_{p} v\left(x_{2}\right)=S_{p} r\left(x_{2}\right)$ since $x_{1}$ and $x_{2}$ visit the same Markov partition elements an equal number of times and $v$ is constant on each partition element. Hence 
it is enough to modify the roof function $\rho: S \rightarrow \mathbb{R}^{+}$in a small neighbourhood of $x_{1}$ that does not intersect the orbit of $x_{2}$ to ensure that the induced roof function $r$ satisfies $S_{p} r\left(x_{1}\right)>S_{p} r\left(x_{2}\right)$ and so is not cohomologous to a piecewise constant roof function. This modification can be done by changing the size of the vector field $X$ in a small neighborhood around $x_{1}$. Note that $S_{p} r\left(x_{i}\right)$ is the period of $x_{i}$ as a periodic orbit of the vector field $X$. By some abuse of notation we shall denote by $X$ the perturbed vector field.

We need to show that this conclusion holds for all vector fields $Y$ that are $C^{k}$ close to $X, k \geq 2$. The orbits of these points involve only finitely many iterates (the inducing time $R$ ) of the Lorenz transformations $f$ : both orbits are contained in $\{R \leq N\}$ for some fixed $N \geq 1$. Moreover, these points belong to two distinct hyperbolic periodic orbits (of saddle type) of the geometric Lorenz attractor and are away from a neighborhood of the singularity at the origin. Hence, these orbits admit a smooth continuation to all $C^{k}$ nearby vector fields $Y$ which admit a similar construction of smooth cross-section $S_{Y}$ and induced tranformation $F_{Y}$, following the inductive procedure detailed in 3 .

In particular, since the periodic points $x_{1}, x_{2}$ belong to the interior of the partition elements we can control finitely many iterates of the Lorenz transformation $f_{Y}$ and obtain that the corresponding partition of $\left\{R_{Y} \leq N\right\}$ associated to the induced transformation $\tilde{F}$ up to inducing time $N$ is close enough to the partition of $\{R \leq N\}$ so that the continuation $\tilde{x}_{i}$ of the orbits of $x_{i}, i=1,2$ have the same combinatorics as before, visiting the same elements of the Markov partition the same number of times as the other but in a different order, and with the same inducing times. The rest of the inducing map for $f_{Y}$ is obtained following an inductive construction and we do not use in this argument the elements of the partition whose induction time is higher than $n$.

We can then use the same expression as before obtaining $S_{p} \tilde{r}\left(\tilde{x}_{1}\right)>S_{p} \tilde{r}\left(\tilde{x}_{2}\right)$ for $Y$ sufficiently $C^{k}$ close to $X$, showing that the induced roof function $\tilde{r}$ for the vector field $Y$ cannot be cohomologous to a piecewise constant roof function. Hence this function satisfies the uniform non-integrability condition needed to obtain exponential decay of correlations for the flow of $Y$ on the geometric Lorenz attractor.

4.3. The hyperbolic skew-product structure. Now we explain how the existence of the previously constructed induced map $F$, together with the existence of the contracting foliation on the cross-section $S$ of the geometric Lorenz flow, ensures the existence of the good hyperbolic skew-product structure for the flow.

We start with a useful consequence of Lemma 3.6 and Remark 3.7 the images of $\Delta$ cover $I$ with the exception of a set of points of zero Lebesgue measure, i.e.

$$
\bigcup_{\omega \in \mathcal{Q}} \bigcup_{j=0}^{R(\omega)-1} f^{j}(\omega)=I, \quad \lambda \bmod 0
$$

In fact, we have that $\Delta_{\mathcal{Q}}:=\cup_{\omega \in \mathcal{Q}} \omega=\Delta \backslash N$ with $\lambda(N)=0$ by construction, and every point of the domain of $f$ has some pre-image in $\Delta$. Since $f^{R(\omega)}(\omega)=\Delta$ for each $\omega \in \mathcal{Q}$ we 
have that

$$
(-1 / 2,1 / 2) \subset \bigcup_{j \geq 0} f^{j}(\Delta)=\bigcup_{j \geq 0} f^{j}(N) \cup \bigcup_{\omega \in \mathcal{Q}}^{R(\omega)-1} \bigcup_{j=0}^{j} f^{j}(\omega)
$$

and $\lambda\left(f^{j}(N)\right)=0$ for all $j \geq 1$ because $f$ is piecewise $C^{2}$, which proves (18).

4.3.1. The induced Poincaré return map. We define the following induced map $\widehat{F}:=P^{R \circ \pi}$ : $\pi^{-1}\left(\Delta_{\mathcal{Q}}\right) \rightarrow \pi^{-1}(\Delta)$, where $\pi: S \rightarrow I$ is the projection onto the quotient $I=S / \mathcal{F}$ of $S$ over the stable leaves. We set for future use $\widehat{\Delta}=\pi^{-1}(\Delta)$ and $\widehat{\Delta}_{\mathcal{Q}}:=\pi^{-1}\left(\Delta_{\mathcal{Q}}\right)=\cup_{\omega \in \mathcal{Q}} \pi^{-1}(\omega)$.

We note that on each element $\pi^{-1}(\omega)$ of the Leb mod 0 partition $\hat{\Delta}_{\mathcal{Q}}$ we have an inducing time given by $R(\omega)$ (we write Leb for a normalized area measure on $S$ ). Moreover by construction $\pi \circ \widehat{F}=F \circ \pi$. The uniform contraction along the leaves of $\mathcal{F}$ ensures that $\widehat{F}$ contracts distances between points in the same leaf. This shows that properties (1) and (4) in Definition 2.3 hold for $\widehat{F}$ on $\widehat{\Delta}$. Properties (2) and (3) will be proven in Subsections 4.3 .3 and 4.4, respectively.

4.3.2. The existence of smooth conjugation. Here we explain how to obtain the smooth semi-conjugacy between the original geometric Lorenz flow model and the hyperbolic skewproduct model.

We can define the semiflow $\widehat{F}_{t}$ over $\widehat{\Delta}$ with base map $\widehat{F}$ and height function $r \circ \pi$ as usual, whose phase space is $\widehat{\Delta}_{r}$ defined in Section 2.3. The suspension semiflow $Z_{t}$ with base map $P: S^{*} \rightarrow S$ and phase space

$$
\Delta_{\tau}:=\left\{(w, t): w \in S^{*}, 0 \leq t<\tau(w)\right\}
$$

can easily seen to be conjugated to the geometric Lorenz flow $Y^{t}$ in a neighborhood of $\Lambda$ through the smooth transformation $\Phi: \Delta_{\tau} \rightarrow U$ given by $\Phi(w, t)=Y^{t}(w)$, where $U$ is a neighborhood of the attractor $\Lambda$ defined in (8). This is a diffeomorphism on an open subset of $U$ with full volume on $M$.

We need to conjugate $Z_{t}$ with $\widehat{F}_{t}$. Since the first return time function $\tau$ is constant on strong-stable leaves, the main diferences between $Z_{t}$ and $\widehat{F}_{t}$ are the base maps and the roof functions. But $F: \Delta_{\mathcal{Q}} \rightarrow \Delta$ and $r$ are induced from $f$ and $\varrho$ with the same number of interates on each $\omega \in \mathcal{Q}$. In fact, since the roof function $r$ associated to $\widehat{F}_{t}$ is an ergodic sum of the roof function $\varrho$ associated to $Z_{t}$ with a locally constant number of summands, which precisely equals $R(\omega)$ on each $\omega \in \mathcal{Q}$, it follows, by the definition of the equivalence relation $\sim$ defining $\Delta_{\tau}$ :

(i) $\widehat{\Delta}_{r}$ can be naturally identified with an open subset of $\Delta_{\tau}$;

(ii) from (18), $\widehat{\Delta}_{r}$ has in fact full bidimensional Lebesgue measure in $\Delta_{\tau}$; and

(iii) $\widehat{F}_{t}(w, s)=Z_{t}(w, s)$ for all $(w, s) \in \widehat{\Delta}_{r}$ and $t \geq 0$.

This shows that we can smoothly conjugate $\widehat{F}_{t}$ with $Z_{t}$ over an open subset with full Lebesgue measure; then smoothly conjugate $Z_{t}$ with the original geometric Lorenz flow $Y^{t}$ on an open subset with full volume in a neighborhood of the attractor $\Lambda$. 
4.3.3. The $\widehat{F}$-invariant probability. In this subsection we use that every invariant measure associated to a quotient map over a stable foliation lifts in a unique way to an invariant measure for the original dynamics to prove item (2) of Definition 2.3.

Let $(S, d)$ be a compact metric space, $\Gamma \subset S$ and let $P: S \backslash \Gamma \rightarrow S$ be a measurable map. We assume that there exists a partition $\mathcal{F}$ of $S$ into measurable subsets, having $\Gamma$ as at most countable collection of elements of $\mathcal{F}$, which is

- invariant: the image by $P$ of any $\xi \in \mathcal{F}$ distinct from $\Gamma$ is contained in some element $\eta$ of $\mathcal{F}$;

- contracting: the diameter of $P^{n}(\xi)$ goes to zero when $n \rightarrow \infty$, uniformly over all the $\xi \in \mathcal{F}$ for which $P^{n}(\xi)$ is defined.

Set $\pi: S \rightarrow \mathcal{F}$ to be the canonical projection. Hence, $A \subset \mathcal{F}$ is Borel measurable if and only if $\pi^{-1}(A)$ is a Borel measurable subset of $S$, since $A$ is open if, and only if, $\pi^{-1}(A)$ is open in $S$. The invariance condition ensures that there is a uniquely defined map

$$
f:(\mathcal{F} \backslash\{\Gamma\}) \rightarrow \mathcal{F} \text { such that } f \circ \pi=\pi \circ P,
$$

which is measurable. We assume that the leaves are sufficiently regular so that $\Xi / \mathcal{F}$ is a metric space with the topology induced by $\pi$.

Let $\mu_{f}$ be any probability measure on $\mathcal{F}$ invariant under the transformation $f$.

For any bounded function $\psi: S \rightarrow \mathbb{R}$, let $\psi_{-}: \mathcal{F} \rightarrow \mathbb{R}$ and $\psi_{+}: \mathcal{F} \rightarrow \mathbb{R}$ be defined by

$$
\psi_{-}(\xi)=\inf _{x \in \xi} \psi(x) \quad \text { and } \quad \psi_{+}(\xi)=\sup _{x \in \xi} \psi(x) .
$$

Proposition 4.8. There exists a unique measure $\mu_{P}$ on $S$ such that

$$
\int \psi d \mu_{P}=\lim _{n \rightarrow \infty} \int\left(\psi \circ P^{n}\right)_{-} d \mu_{f}=\lim _{n \rightarrow \infty} \int\left(\psi \circ P^{n}\right)_{+} d \mu_{f}
$$

for every continuous function $\psi: S \rightarrow \mathbb{R}$. Besides, $\mu_{P}$ is invariant under $P$. Moreover the correspondence $\mu_{f} \mapsto \mu_{P}$ is injective, $\pi_{*} \mu_{P}=\mu_{f}$ and $\mu_{P}$ is ergodic if $\mu_{f}$ is ergodic.

This follows from standard arguments which can be found in, e.g. Section 7.3.5 of [7]. Hence we just have to take $\mu_{f}=\nu_{0}$ to obtain the corresponding $\eta_{0}=\mu_{P}$ ergodic $P$ invariant probability measure which lifts $\nu_{0}$, where $P$ and $f$ are the Poincaré return map to the cross-section $S$ of the geometric Lorenz flow, $f$ the Lorenz transformation associated to $P$; and $\mathcal{F}$ is the family of stable leaves on $S$ for $P$.

Analogously, we consider the measurable map $\widehat{F}: \widehat{\Delta}_{\mathcal{Q}} \rightarrow \widehat{\Delta}$ on the space $\widehat{\Delta}$ with the same foliation $\mathcal{F}$ of $S$ restricted to $\widehat{\Delta}$, together with the quotient map $F: \Delta_{\mathcal{Q}} \rightarrow \Delta$. Then we start with the $F$-invariant ergodic measure $\nu$ and obtain an $\widehat{F}$-invariant ergodic measure $\eta$ on $\widehat{\Delta}$.

4.4. The disintegration property. Here we show that the previous measure $\eta$ admits a smooth disintegration as stated at item (3) of Definition 2.3 under the following assumptions on $\mathcal{F}$ and $f$, besides invariance and contraction as in the previous subsection:

- $S / \mathcal{F}$ is the compact closure of an open domain of a finite dimensional smooth manifold; 
- $f: \mathcal{F} \backslash \Gamma \rightarrow \mathcal{F}$ is a uniformly expanding Markov map, according to Section 2.1,

- The invariant density $\phi=d \mu_{f} / d$ Leb is a $C^{1}$ function.

We note that the assumption of denumerability of $\Gamma$ ensures that $S \backslash \Gamma$ is $\sigma$-compact.

The general strategy of the argument is to obtain the disintegration of $\eta$ as fixed point of a certain transfer operator whose action from fiber to fiber varies differentiably.

To avoid the introduction of extra notation and to focus on the the geometric Lorenz attractor case, from now on we take $\widehat{F}: \widehat{\Delta}_{\mathcal{Q}} \rightarrow \widehat{\Delta}$ on the space $\widehat{\Delta}$ with the foliation $\mathcal{F}$ of $S$ restricted to $\widehat{\Delta}$, together with the quotient map $F: \Delta_{\mathcal{Q}} \rightarrow \Delta$, as our main maps. Let us consider the set $\Omega$ of measurable families of probability measures $\omega=\left(\omega_{x}\right)_{x \in I}$ supported on the strong-stable leaves $\left\{\pi^{-1}(x) \cap \Lambda\right\}_{x \in I}$ inside the geometric Lorenz attractor $\Lambda$. We note that $I \ni x \mapsto \omega_{x}$ is measurable if the real function $x \mapsto \int \psi d \omega_{x}$ is measurable for every continuous function $\psi: \widehat{\Delta}_{\mathcal{Q}} \rightarrow \mathbb{R}$ with compact support. Each such family defines a probability measure $\widetilde{\nu^{\omega}}$ through the linear functional

$$
C_{0}^{0}\left(\widehat{\Delta}_{\mathcal{Q}}, \mathbb{R}\right) \ni \psi \mapsto \iint \psi d \omega_{x} d \nu(x)
$$

where $C_{0}^{0}\left(\widehat{\Delta}_{\mathcal{Q}}, \mathbb{R}\right)$ is the set of all continuous functions on $\widehat{\Delta}_{\mathcal{Q}}$ with compact support; see e.g. [20] for the definition and properties of Radon measures. We define the operator $\mathcal{L}: \Omega \rightarrow \Omega$ such that

$$
\iint \psi d \mathcal{L}(\omega)_{x} d \nu(x)=\iint \psi \circ \widehat{F} d \omega_{x} d \nu(x), \quad \psi \in C_{0}^{0}\left(\widehat{\Delta}_{\mathcal{Q}}, \mathbb{R}\right) .
$$

This is the dual of the usual Koopman operator $U: C_{0}^{0}\left(\widehat{\Delta}_{\mathcal{Q}}, \mathbb{R}\right) \rightarrow C_{0}^{0}\left(\widehat{\Delta}_{\mathcal{Q}}, \mathbb{R}\right)$ given by $U(\psi)=\psi \circ \widehat{F}$. Moreover, it defines an operator using the disintegration of the measure $\widetilde{\nu^{\omega}}$ defined by the linear functional

$$
C_{0}^{0}\left(\widehat{\Delta}_{\mathcal{Q}}, \mathbb{R}\right) \ni \psi \mapsto \iint \psi \circ \widehat{F} d \omega_{x} d \nu(x)
$$

with respect to the measurable partition of $\widehat{\Delta}$ given by the restriction of $\mathcal{F}$ to this set. This defines the family $(\mathcal{L}(\omega))_{x}$ for $\nu$-almost every $x \in I$ in a unique way; see e.g. [41] for more on disintegration with respect to measurable partitions of Lebesgue spaces. The next lemma establishes that invariant measures arise as fixed points for $\mathcal{L}$. More precisely,

Lemma 4.9. Given $\omega \in \Omega$ and $\psi \in C_{0}^{0}\left(\widehat{\Delta}_{\mathcal{Q}}, \mathbb{R}\right)$ there exists the limit

$$
\nu^{\bar{\omega}}(\psi):=\lim _{n}\left(\mathcal{L}^{n} \omega\right) \psi=\lim _{n} \iint \psi \circ \widehat{F}^{n} d \omega_{x} d \nu(x) .
$$

Moreover, the probability measure $\nu^{\bar{\omega}}$ is $\widehat{F}$-invariant and the family $\bar{\omega}$ does not depend on $\omega$ on $\nu$-almost every point; in fact $\nu^{\bar{\omega}}=\eta$.

Proof. Let $\psi \in C_{0}^{0}\left(\widehat{\Delta}_{\mathcal{Q}}, \mathbb{R}\right)$ and $\omega \in \Omega$ be fixed. Given $\varepsilon>0$, let $\delta>0$ be such that $|\psi(A)-\psi(B)| \leq \varepsilon$ for all $A, B \subset S$ with $\operatorname{dist}(A, B) \leq \delta$, where dist denotes the euclidean distance. Since the partition $\mathcal{F}$ is assumed to be contracting, there exists $n_{0} \geq 0$ such that 
$\operatorname{diam}\left(\widehat{F}^{n}(\xi)\right) \leq \delta$ for every $\xi \in \mathcal{F}$ and any $n \geq n_{0}$. Let $n+k \geq n \geq n_{0}, A \subset \pi^{-1}\{x\}$ and $B \subset \pi^{-1}\left\{f^{k}(x)\right\}$. Then

$$
\left|\psi \circ \widehat{F}^{n+k}(A)-\psi \circ \widehat{F}^{n}(B)\right| \leq \sup \left(\psi \mid \widehat{F}^{n+k}\left(\pi^{-1}\{x\}\right)\right)-\inf \left(\psi \mid \widehat{F}^{n}\left(\pi^{-1}\left\{F^{k}(x)\right\}\right)\right) \leq \varepsilon
$$

since $\widehat{F}^{n+k}\left(\pi^{-1}\{x\}\right) \subset \widehat{F}^{n}\left(\pi^{-1}\left\{F^{k}(x)\right\}\right)$. Thus, using the $F^{k}$-invariance of $\nu$ we get from the previous estimate that

$$
\begin{aligned}
\left|\left(\mathcal{L}^{n+k} \omega\right) \psi-\left(\mathcal{L}^{n} \omega\right) \psi\right| & =\left|\iint \psi \circ \widehat{F}^{n+k} d \omega_{x} d \nu(x)-\iint \psi \circ \widehat{F}^{n} d \omega_{x} d \nu(x)\right| \\
& =\left|\iint \psi \circ \widehat{F}^{n+k} d \omega_{x} d \nu(x)-\iint \psi \circ \widehat{F}^{n} d \omega_{F^{k}(x)} d \nu(x)\right| \\
& \leq \int\left|\int \psi \circ \widehat{F}^{n+k} d \omega_{x}-\int \psi \circ \widehat{F}^{n} d \omega_{F^{k}(x)}\right| d \nu(x) \leq \varepsilon .
\end{aligned}
$$

This shows that the sequence $\left(\mathcal{L}^{n} \omega\right) \psi$ is a Cauchy sequence and so it converges in the Banach space $C_{0}^{0}\left(\widehat{\Delta}_{\mathcal{Q}}, \mathbb{R}\right)$. It is straightforward to check that, since each element of the sequence is a normalized positive linear functional, the limit is a family $\bar{\omega} \in \Omega$ such that $\nu^{\bar{\omega}}$ has the same functional properties, and so represents a probability measure. We remark that for any compact subset $K$ of $\widehat{\Delta}$ we can rewrite the last inequality above as follows

$$
\int\left|\int_{K} \psi \circ \widehat{F}^{n+k} d \omega_{x}-\int_{K} \psi \circ \widehat{F}^{n} d \omega_{F^{k}(x)}\right| d \nu(x) \leq \varepsilon .
$$

This shows that the convergence does not depend on the support of $\psi \circ \widehat{F}^{n}$ for arbitrarily large $n$. Note also that given $\varphi \in C_{0}^{0}\left(\Delta_{\mathcal{Q}}, \mathbb{R}\right)$ the function $\varphi \circ \pi$ belongs to $C_{0}^{0}\left(\widehat{\Delta}_{\mathcal{Q}}, \mathbb{R}\right)$ and is constant on each leaf of $\mathcal{F}$ through $\widehat{\Delta}$, which yields

$$
\nu^{\bar{\omega}}(\varphi \circ \pi)=\lim _{n} \iint \varphi \circ \pi \circ \widehat{F}^{n} d \omega_{x} d \nu(x)=\lim _{n} \int \varphi\left(F^{n}(x)\right) d \nu(x)=\int \varphi d \nu,
$$

that is $\pi_{*}\left(\nu^{\bar{\omega}}\right)=\nu$. Hence, if we show that $\nu^{\bar{\omega}}$ is $\widehat{F}$-invariant, we can use Proposition 4.8 to conclude that $\eta=\nu^{\bar{\omega}}$ independently of the starting family $\omega \in \Omega$. To prove invariance, we observe that

$$
\left(\mathcal{L}^{n+1} \omega\right) \psi=\mathcal{L}\left(\mathcal{L}^{n} \omega\right) \psi=\iint \psi \circ \widehat{F} d\left(\mathcal{L}^{n}(\omega)_{x}\right) d \nu(x)
$$

but $\psi \circ \widehat{F}$ is not continuous with compact support for $\psi \in C_{0}^{0}\left(\widehat{\Delta}_{\mathcal{Q}}, \mathbb{R}\right)$. From (21) we have that, using the $\sigma$-compactness of $\widehat{\Delta}_{\mathcal{Q}}$ and choosing a nested increasing sequence $K_{l}$ of compact sets growing to $\widehat{\Delta}$ and a non-negative $\psi \in C_{0}^{0}\left(\widehat{\Delta}_{\mathcal{Q}}, \mathbb{R}\right)$, we get for $n \geq n_{0}, m \geq$ $1, l \geq 1$

$$
\left|\iint_{K_{l}} \psi \circ \widehat{F} d\left(\mathcal{L}^{n} \omega\right)_{x} d \nu(x)-\iint_{K_{l}} \psi \circ \widehat{F} d\left(\mathcal{L}^{n+m} \omega\right)_{x} d \nu(x)\right| \leq \varepsilon
$$


To ensure that $\psi \circ \widehat{F} \mid K_{l}$ is continuous with compact support, we observe that

$$
\operatorname{supp}(\psi \circ \widehat{F})=\widehat{F}^{-1}(\operatorname{supp} \psi)=\bigcup_{\omega \in \mathcal{Q}}\left(P^{R(\omega)}\right)^{-1}(\operatorname{supp} \psi)
$$

is a denumerable union of compacts in $\widehat{\Delta}$, because supp $\psi$ is compact and $P: S^{*} \rightarrow S$ is a diffeomorphism onto its image. Thus we can choose an enumeration $\left\{\omega_{n}\right\}_{n \geq 1}$ of $\mathcal{Q}$ and define

$$
K_{l}:=\bigcup_{i=1}^{l}\left(P^{R\left(\omega_{i}\right)}\right)^{-1}(\operatorname{supp} \psi)
$$

to obtain a sequence such that $\psi \circ \widehat{F} \mid K_{l} \nearrow \psi \circ \widehat{F}$ is a monotonous sequence of continuous functions of compact support. Hence, letting $m$ grow without bound in (22) we arrive at

$$
\left|\iint_{K_{l}} \psi \circ \widehat{F} d\left(\mathcal{L}^{n} \omega\right)_{x} d \nu(x)-\iint_{K_{l}} \psi \circ \widehat{F} d \bar{\omega}_{x} d \nu(x)\right| \leq \varepsilon .
$$

Making $l$ grow we finally obtain $\left|\mathcal{L}^{n+1} \psi-\mathcal{L}(\bar{\omega}) \psi\right| \leq \varepsilon$ for $n \geq n_{0}$. These arguments assumed that $\psi$ is non-negative; but for a continuous function with compact support, we can write $\psi=\psi^{+}-\psi^{-}$with $\psi^{ \pm}$non-negative and still continuous, and then apply the same argument to each summand using linearity.

Finally, $\varepsilon>0$ can be arbitrarily chosen at the start, so we have proved that

$$
\bar{\omega}=\lim _{n} \mathcal{L}^{n} \omega=\mathcal{L}(\bar{\omega}) \quad \text { which implies } \quad \nu^{\bar{\omega}}=\widehat{F}_{*}\left(\nu^{\bar{\omega}}\right)
$$

as needed to complete the uniqueness part of the statement.

4.4.1. Conditional measures as uniform limits. To prove that $\bar{u}(x):=\int u(x, y) d \bar{\omega}_{x}(y)$ is a $C^{1}$ map with bounded derivative, for each $C^{1}$ function $u: \widehat{\Delta}_{\mathcal{Q}} \rightarrow \mathbb{R}$ with compact support, we need some preliminary results. We can be more precise about the operator $\mathcal{L}$ in the next proposition, whose proof will be given later in this section.

Proposition 4.10. For every bounded measurable function $\psi: \widehat{\Delta}_{\mathcal{Q}} \rightarrow \mathbb{R}$ and for the constant family $v=\left(v_{x}\right) \in \Omega$ with $v_{x}=\left.\lambda\right|_{\pi^{-1}(\{x\})}$ for each $x \in I$, we have for $\lambda$-almost every $x \in I$

$$
\int \psi d\left(\mathcal{L}^{n} v\right)_{x}=\sum_{h \in \mathcal{H}_{n}} \int \frac{\phi \cdot \psi \circ \widehat{F}_{t}^{n}}{\phi \circ F^{n} \cdot D F^{n}}(h(x)) d \lambda(t), \quad \text { for each } n \geq 1
$$

where $\phi:=d \nu / d \lambda$ is the Hölder-continuous density of $\nu$ with respect to $\lambda$; and we write, to simplify the notation, $\widehat{F}_{t}^{n}(z):=\widehat{F}^{n}(z, t)$ for $(z, t) \in \widehat{\Delta}_{\mathcal{Q}}$.

Remark 4.11. Note that $D F>0$ and so it is useful to write the transfer or Ruelle-PerronFrobenius operator associated to $F$ and the potential $-\log |D F|$ as

$$
\mathcal{P}(\varphi)(x):=\sum_{h \in \mathcal{H}_{1}} \frac{1}{D F(h(x))} \varphi(h(x)) .
$$


Hence, $\mathcal{P} \phi=\phi$ and

$$
\sum_{h \in \mathcal{H}_{n}} \frac{\phi \cdot \psi \circ \widehat{F}_{t}^{n}}{\phi \circ F^{n} \cdot D F^{n}} \circ h=\frac{1}{\phi} \sum_{h \in \mathcal{H}_{n}} \frac{\phi \cdot \psi \circ \widehat{F}_{t}^{n}}{D F^{n}} \circ h=\frac{1}{\phi} \mathcal{P}^{n}\left(\phi \cdot \psi \circ \widehat{F}_{t}^{n}\right) .
$$

From Lemma 4.9 we can obtain the invariant family $\bar{\omega}$ as the limit of $\left(\mathcal{L}^{n} v\right)_{n \geq 1}$, so Proposition 4.10 provides an explicit expression to approximate the elements of $\bar{\omega}$. The proof of this proposition becomes simpler if we use the following lemmas.

Lemma 4.12. For every fixed $n \in \mathbb{Z}^{+}$, every $t \in\left[-\frac{1}{2}, \frac{1}{2}\right]$ and each bounded measurable function $\psi: \widehat{\Delta}_{\mathcal{Q}} \rightarrow \mathbb{R}$, the series given by $\frac{1}{\phi} \mathcal{P}^{n}\left(\phi \cdot \psi \circ \widehat{F}_{t}^{n}\right)$ is absolutely convergent Lebesgue almost everywhere.

Proof. Indeed, since $\phi$ is a $C^{1}$ function bounded from above and below (see Subsection 4.1.1 and Lemma 4.6) there exists $C>0$, depending only on $\phi$, such that for $\lambda$-almost every $x \in I$

$$
\left|\sum_{h \in \mathcal{H}_{n}} \frac{\phi \cdot \psi \circ \widehat{F}_{t}^{n}}{\phi \circ F^{n} \cdot D F^{n}}(h(x))\right| \leq \sum_{h \in \mathcal{H}_{n}} \frac{C \cdot\|\psi\|_{\infty}}{D F^{n}(h(x))} \leq \sum_{h \in \mathcal{H}_{n}} \frac{C \cdot \lambda(h(\Delta))}{\lambda(\Delta)}<\infty
$$

since $\psi$ is essentially bounded, $\{h(\Delta)\}_{h \in \mathcal{H}_{n}}=\mathcal{Q}$ is a partition of $\Delta$ Lebesgue modulo zero, and by the bounded distortion property combined with the mean value theorem. Indeed, from Proposition 4.7 we have for every $h \in \mathcal{H}_{n}, x \in \Delta$ and some $z=z(h) \in h(\Delta)$

$$
\lambda(\Delta)=\lambda(h(\Delta)) D F^{n}(h(z)) \leq B_{0} \cdot \lambda(h(\Delta)) D F^{n}(h(x))
$$

and $B_{0}$ does not depend on $n \geq 1$.

From the previous argument we obtain a useful property for the transfer operator $\mathcal{P}$ for the expanding map $F$, to be used in what follows.

Lemma 4.13. The spectral radius of the operator $\mathcal{P}: L^{\infty}(I, \lambda) \rightarrow L^{\infty}(I, \lambda)$ is equal to 1 .

Proof. We note that the operator is well defined on essentially bounded functions by the previous lemma. Since $\mathcal{P}(\phi)=\phi$ the spectral radius is at least 1 . Taking a bounded measurable function $\psi: \widehat{\Delta}_{\mathcal{Q}} \rightarrow \mathbb{R}$ we can write

$$
\left|\mathcal{P}^{n}(\psi)(x)\right|=\left|\sum_{h \in \mathcal{H}_{n}} \frac{1}{D F^{n}(h(x))} \psi(h(x))\right| \leq\|\psi\|_{\infty} \mathcal{P}^{n}(1) \leq B_{0} \cdot\|\psi\|_{\infty}
$$

for Lebesgue almost every $x \in I$ and every $n \geq 1$, using the relation (23). Hence the spectral radius verifies $\operatorname{sp}(\mathcal{P})=\lim \sup \sqrt[n]{\left\|\mathcal{P}^{n}\right\|} \leq \lim \sqrt[n]{B_{0}}=1$.

Lemma 4.14. The sequence $\left(\frac{1}{\phi} \mathcal{P}^{n}\left(\phi \cdot \psi \circ \widehat{F}_{t}^{n}\right)\right)_{n \geq 1}$ is uniformly convergent in $(x, t) \in \Delta \times I$ for each continuous function $\psi: \widehat{\Delta}_{\mathcal{Q}} \rightarrow \mathbb{R}$ with compact support. 
Proof. We show that the sequence is a uniform Cauchy sequence. Let us fix $\varepsilon>0$, then $\psi$ as in the statement and take $n_{0}$ as in (20) and $n+k>n \geq n_{0}$. If we fix $(x, t) \in \Delta \times I$, then

$$
\begin{aligned}
\sum_{h \in \mathcal{H}_{n}} \frac{\phi \cdot \psi \circ \widehat{F}_{t}^{n}}{D F^{n}} \circ h & -\sum_{h \in \mathcal{H}_{n+k}} \frac{\phi \cdot \psi \circ \widehat{F}_{t}^{n+k}}{D F^{n+k}} \circ h=\sum_{h \in \mathcal{H}_{n}}\left(\frac{\phi \cdot \psi \circ \widehat{F}_{t}^{n}}{D F^{n}}-\sum_{\ell \in \mathcal{H}_{k}} \frac{\phi \cdot \psi \circ \widehat{F}_{t}^{n+k}}{\left.D F^{n+k} \circ \ell\right) \circ h}\right. \\
& =\sum_{h \in \mathcal{H}_{n}}\left(\frac{\phi \cdot \psi \circ \widehat{F}_{t}^{n}}{D F^{n}}-\sum_{\ell \in \mathcal{H}_{k}} \frac{\phi \cdot \psi \circ \widehat{F}_{t}^{n+k}}{D F^{n} \circ F^{k} \cdot D F^{k}} \circ \ell\right) \circ h \\
& =\sum_{h \in \mathcal{H}_{n}} \frac{1}{D F^{n}}\left(\phi \cdot \psi \circ \widehat{F}_{t}^{n}-\sum_{\ell \in \mathcal{H}_{k}} \frac{\phi \cdot \psi \circ \widehat{F}_{t}^{n+k}}{D F^{k}} \circ \ell\right) \circ h .
\end{aligned}
$$

Defining $\Delta_{\ell}^{n}:=\psi \circ \widehat{F}_{t}^{n}-\psi \circ \widehat{F}_{t}^{n+k} \circ \ell$ we can rewrite the above as

$$
\begin{aligned}
\sum_{h \in \mathcal{H}_{n}} & \frac{1}{D F^{n}}\left(\phi \cdot \psi \circ \widehat{F}_{t}^{n}-\sum_{\ell \in \mathcal{H}_{k}} \frac{\phi \cdot \psi \circ \widehat{F}_{t}^{n}}{D F^{k}} \circ \ell+\sum_{\ell \in \mathcal{H}_{k}} \frac{\phi}{D F^{k}} \circ \ell \cdot \Delta_{\ell}^{n}\right) \circ h \\
= & \sum_{h \in \mathcal{H}_{n}} \frac{1}{D F^{n}}[\psi \circ \widehat{F}_{t}^{n} \underbrace{\left(\phi-\sum_{\ell \in \mathcal{H}_{k}} \frac{\phi}{D F^{k}} \circ \ell\right)}_{\phi-\mathcal{P}(\phi)=0}+\sum_{\ell \in \mathcal{H}_{k}} \frac{\phi}{D F^{k}} \circ \ell \cdot \Delta_{\ell}^{n}] \circ h \\
= & \sum_{h \in \mathcal{H}_{n}} \frac{1}{D F^{n}} \circ h \cdot\left(\sum_{\ell \in \mathcal{H}_{k}} \frac{\phi}{D F^{k}} \circ \ell \cdot \Delta_{\ell}^{n}\right) \circ h=\sum_{h \in \mathcal{H}_{n+k}}\left(\frac{\phi}{D F^{n+k}} \circ h\right) \cdot\left(\Delta_{\ell}^{n} \circ F^{k} \circ h\right)
\end{aligned}
$$

Using $\phi>0$ and (20) it follows that absolute value of the last expression is bounded by

$$
\sum_{h \in \mathcal{H}_{n+k}}\left|\Delta_{\ell}^{n} \circ F^{k} \circ h\right| \cdot\left(\frac{\phi}{D F^{n+k}} \circ h\right) \leq \varepsilon \sum_{h \in \mathcal{H}_{n+k}} \frac{\phi}{D F^{n+k}} \circ h=\varepsilon \phi .
$$

Finally, since $\varepsilon>0$ and $(x, t) \in \Delta \times I$ were arbitrarily chosen the proof is complete.

Proof of Proposition 4.10. We fix $n=1$ for definiteness since the general case of $n>1$ is completely analogous. From Lemma 4.12 the series $\frac{1}{\phi} \mathcal{P}^{n}\left(\phi \cdot \psi \circ \widehat{F}_{t}^{n}\right)$ is absolutely convergent. 
Hence we can exchange the integral and the summation and apply a change of variables

$$
\begin{aligned}
\iint \psi d(\mathcal{L} v)_{x}(t) d \nu(x) & =\int_{\Delta} \int \psi \circ \widehat{F} d v_{x} d \nu=\sum_{h \in \mathcal{H}_{1}} \int_{h(\Delta)} \int \psi \circ \widehat{F}_{t}(x) d\left(v_{x}\right)(t) d \nu(x) \\
& =\sum_{h \in \mathcal{H}_{1}} \int_{h(\Delta)} \int \phi(x) \cdot \psi \circ \widehat{F}_{t}(x) d \lambda(t) d \lambda(x) \\
& =\sum_{h \in \mathcal{H}_{1}} \int_{h(\Delta)}\left(\int \frac{\phi \cdot \psi \circ \widehat{F}_{t}}{\phi \circ F \cdot D F}(h(F(x))) d \lambda(t)\right) D F(x) \phi(F(x)) d \lambda(x) \\
& =\sum_{h \in \mathcal{H}_{1}} \int_{F(h(\Delta))}\left(\int \frac{\phi \cdot \psi \circ \widehat{F}_{t}}{\phi \circ F \cdot D F}(h(x)) d \lambda(t)\right) \phi(x) d \lambda(x) \\
& =\int \sum_{h \in \mathcal{H}_{1}} \int \frac{\phi \cdot \psi \circ \widehat{F}_{t} \circ h d \lambda(t) d \nu(x)}{\phi \circ F \cdot D F}
\end{aligned}
$$

and the statement of the lemma follows from the uniqueness of the disintegration.

At this point we note that $L(\psi)_{x}:=\lim _{n \rightarrow+\infty} \int \psi d\left(\mathcal{L}^{n} v\right)_{x}$ is clearly a normalized, positive and bounded linear functional on $C_{0}^{0}\left(\Delta_{\mathcal{Q}}, \mathbb{R}\right)$, thus there exists a probability measure $\widetilde{\omega}_{x}$ such that $L(\psi)_{x}=\int \psi d \widetilde{\omega}_{x}$. Hence, since we have uniform convergence in Lemma 4.14

$$
\lim _{n \rightarrow+\infty} \iint \psi d\left(\mathcal{L}^{n} v\right)_{x} d \nu(x)=\int \lim _{n \rightarrow+\infty} \int \psi d\left(\mathcal{L}^{n} v\right)_{x} d \nu(x)=\iint \psi d \widetilde{\omega}_{x} d \nu(x)
$$

and because from Lemma 4.9 we also have

$$
\lim _{n \rightarrow+\infty} \iint \psi d\left(\mathcal{L}^{n} v\right)_{x} d \nu(x)=\iint \psi d \bar{\omega}_{x} d \nu(x)
$$

for each continuous function with compact support, the uniqueness of disintegration ensures that $\bar{\omega}_{x}=\widetilde{\omega}_{x}=\lim _{n \rightarrow+\infty}\left(\mathcal{L}^{n} v\right)_{x}$ for $\nu$-almost every point $x$. Since we need to establish the smoothness of the disintegration we define

$$
\bar{\omega}_{x}:=\lim _{n \rightarrow+\infty}\left(\mathcal{L}^{n} v\right)_{x} \quad \text { for all } x \in \Delta
$$

and study in more detail this limit process.

4.4.2. Disintegration is smooth. We fix $u \in C_{0}^{1}\left(\Delta_{\mathcal{Q}}, \mathbb{R}\right)$. From Proposition 4.10 we have

$$
\bar{u}(x)=\int u(x, t) d \bar{\omega}_{x}(t)=\int \lim _{n \rightarrow+\infty} \frac{1}{\phi} \mathcal{P}^{n}\left(\phi \cdot u \circ \widehat{F}_{t}^{n}\right)(x) d \lambda(t) .
$$

We shall prove that there is a well defined limit for the expression of the derivative $D\left[\frac{1}{\phi} \mathcal{P}^{n}\left(\phi \cdot u \circ \widehat{F}_{t}^{n}\right)\right]$ as $n \rightarrow \infty$ with uniform bounds independently of $t$. In fact, by a straightforward computation using the chain rule and $\left|h^{\prime}(x)\right|=\left|D F^{n}(h(x))\right|^{-1}$ for every 
$h \in \mathcal{H}_{n}$ we get, for every $x \in \Delta$

$$
\begin{aligned}
D\left[\frac{1}{\phi(x)} \mathcal{P}^{n}\left(\phi \cdot u \circ \widehat{F}_{t}^{n}\right)(x)\right] & =-\frac{D \phi(x)}{\phi(x)^{2}} \mathcal{P}^{n}\left(\phi \cdot u \circ \widehat{F}_{t}^{n}\right)(x) \\
& -\frac{1}{\phi(x)} \sum_{h \in \mathcal{H}_{n}}\left(\frac{D^{2} F^{n}}{\left(D F^{n}\right)^{2}} \cdot \frac{\phi \cdot u \circ \widehat{F}_{t}^{n}}{D F^{n}}\right) \circ h(x) \\
& +\frac{1}{\phi(x)} \sum_{h \in \mathcal{H}_{n}}\left(\frac{1}{D F^{n}} \cdot \frac{D\left(\phi \cdot u \circ \widehat{F}_{t}^{n}\right)}{D F^{n}}\right) \circ h(x) .
\end{aligned}
$$

Since $\left|D F^{n}\right| \geq \sigma^{-n}=(\sqrt{2})^{n}$ and $\mathcal{P}$ is a positive operator with spectral radius equal to one from Lemma 4.13, we see that the absolute value of (26) is bounded from above by $\frac{1}{\inf \phi} \sigma^{n}\left\|\mathcal{P}^{n}(1)\right\|_{\infty}\|\phi \cdot u\|_{C^{1}}$, and so converges to zero.

Moreover, using the consequence (13) of the Renyi condition from Subsection 4.1.1, we

deduce that the absolute value of (25) is bounded from above by $B n \sigma^{n-1} \frac{1}{\phi(x)} \mathcal{P}^{n}\left(\phi \cdot u \circ \widehat{F}_{t}^{n}\right)(x)$ and so, using Lemma 4.14, it converges uniformly to zero as $n \rightarrow \infty$. Hence, we get that

$$
D \bar{u}(x)=-\frac{D \phi(x)}{\phi(x)} \int \lim _{n \rightarrow+\infty} \frac{1}{\phi} \mathcal{P}^{n}\left(\phi \cdot u \circ \widehat{F}_{t}^{n}\right) d \lambda(t)=-\frac{D \phi(x)}{\phi(x)} \bar{u}(x)
$$

and so $D \bar{u}$ exists, hence $\bar{u}$ is continuous, thus by the last identity $D \bar{u}$ is also continuous. This proves that the disintegration $\left(\bar{\omega}_{x}\right)_{x}$ is smooth.

Remark 4.15. The differential equation above has a solution $\bar{u}(x)=\frac{1}{\phi(x)}+c(t)$. But since

$$
\int u d \eta=\int \bar{u} d \nu=\int(1+c(t) \phi(x)) d \lambda(x)=1+c(t)
$$

we see that $c(t) \equiv c(u)=\int u d \eta-1$.

\section{REFERENCES}

[1] V. S. Afraimovich, V. V. Bykov, and L. P. Shil'nikov. On the appearence and structure of the Lorenz attractor. Dokl. Acad. Sci. USSR, 234:336-339, 1977.

[2] J. F. Alves, C. Bonatti, and M. Viana. SRB measures for partially hyperbolic systems whose central direction is mostly expanding. Invent. Math., 140(2):351-398, 2000.

[3] J. F. Alves, S. Luzzatto, and V. Pinheiro. Lyapunov exponents and rates of mixing for one-dimensional maps. Ergodic Theory Dynam. Systems, 24(3):637-657, 2004.

[4] V. Araújo. Large deviations bound for semiflows over a non-uniformly expanding base. Bull. Braz. Math. Soc. (N.S.), 38(3):335-376, 2007.

[5] V. Araujo, S. Galatolo, and M. J. Pacifico. Decay of correlations of maps with uniformly contracting fibers and logarithm law for singular hyperbolic attractors. In preparation, 2011.

[6] V. Araújo and M. J. Pacifico. Large deviations for non-uniformly expanding maps. J. Stat. Phys., $125(2): 415-457,2006$.

[7] V. Araújo and M. J. Pacifico. Three-dimensional flows, volume 53 of Ergebnisse der Mathematik und ihrer Grenzgebiete. 3. Folge. A Series of Modern Surveys in Mathematics [Results in Mathematics and Related Areas. 3rd Series. A Series of Modern Surveys in Mathematics]. Springer, Heidelberg, 2010. With a foreword by Marcelo Viana. 
[8] V. Araújo, E. R. Pujals, M. J. Pacifico, and M. Viana. Singular-hyperbolic attractors are chaotic. Transactions of the A.M.S., 361:2431-2485, 2009.

[9] A. Avila, S. Gouëzel, and J.-C. Yoccoz. Exponential mixing for the Teichmüller flow. Publ. Math. Inst. Hautes Études Sci., 104:143-211, 2006.

[10] V. Baladi and B. Vallée. Exponential decay of correlations for surface semi-flows without finite Markov partitions. Proc. Amer. Math. Soc., 133(3):865-874, 2005.

[11] C. Bonatti, A. Pumariño, and M. Viana. Lorenz attractors with arbitrary expanding dimension. $C$. R. Acad. Sci. Paris Sér. I Math., 325(8):883-888, 1997.

[12] R. Bowen. Equilibrium states and the ergodic theory of Anosov diffeomorphisms, volume 470 of Lect. Notes in Math. Springer Verlag, 1975.

[13] R. Bowen and D. Ruelle. The ergodic theory of Axiom A flows. Invent. Math., 29:181-202, 1975.

[14] A. I. Bufetov. Decay of correlations for the Rauzy-Veech-Zorich induction map on the space of interval exchange transformations and the central limit theorem for the Teichmüller flow on the moduli space of abelian differentials. J. Amer. Math. Soc., 19(3):579-623, 2006.

[15] N. I. Chernov. Markov approximations and decay of correlations for Anosov flows. Ann. of Math. (2), 147(2):269-324, 1998.

[16] P. Collet, H. Epstein, and G. Gallavotti. Perturbations of geodesic flows on surfaces of constant negative curvature and their mixing properties. Comm. Math. Phys., 95(1):61-112, 1984.

[17] K. Díaz-Ordaz. Decay of correlations for non-Hölder observables for one-dimensional expanding Lorenz-like maps. Discrete Contin. Dyn. Syst., 15(1):159-176, 2006.

[18] D. Dolgopyat. On decay of correlations in Anosov flows. Ann. of Math. (2), 147(2):357-390, 1998.

[19] D. Dolgopyat. Prevalence of rapid mixing in hyperbolic flows. Ergodic Theory Dynam. Systems, 18(5):1097-1114, 1998.

[20] L. C. Evans and R. F. Gariepy. Measure theory and fine properties of functions. Studies in Advanced Mathematics. CRC Press, 1992.

[21] M. Field, I. Melbourne, and A. Törok. Stability of mixing and rapid mixing for hyperbolic flows. Annals of Mathematics, 166:269-291, 2007.

[22] S. Galatolo and M. J. Pacifico. Lorenz like flows: exponential decay of correlations for the poincaré map, logarithm law, quantitative recurrence. Ergodic Theory and Dynamical Systems, 30:703-1737, Jan. 2010.

[23] S. Gouëzel. Decay of correlations for nonuniformly expanding systems. Bull. Soc. Math. France, 134(1):1-31, 2006.

[24] J. Guckenheimer and R. F. Williams. Structural stability of Lorenz attractors. Publ. Math. IHES, 50:59-72, 1979.

[25] P. Hartman. Ordinary differential equations, volume 38 of Classics in Applied Mathematics. Society for Industrial and Applied Mathematics (SIAM), Philadelphia, PA, 2002. Corrected reprint of the second (1982) edition [Birkhäuser, Boston, MA; MR0658490 (83e:34002)], With a foreword by Peter Bates.

[26] M. Hirsch, C. Pugh, and M. Shub. Invariant manifolds, volume 583 of Lect. Notes in Math. Springer Verlag, New York, 1977.

[27] M. Holland and I. Melbourne. Central limit theorems and invariance principles for Lorenz attractors. J. Lond. Math. Soc. (2), 76(2):345-364, 2007.

[28] C. Liverani. On contact Anosov flows. Ann. of Math. (2), 159(3):1275-1312, 2004.

[29] E. N. Lorenz. Deterministic nonperiodic flow. J. Atmosph. Sci., 20:130-141, 1963.

[30] S. Luzzatto, I. Melbourne, and F. Paccaut. The Lorenz attractor is mixing. Comm. Math. Phys., 260(2):393-401, 2005.

[31] I. Melbourne and A. Török. Central limit theorems and invariance principles for time-one maps of hyperbolic flows. Comm. Math. Phys., 229(1):57-71, 2002. 
[32] I. Melbourne and A. Török. Statistical limit theorems for suspension flows. Israel J. Math., 144:191209, 2004.

[33] R. Metzger and C. Morales. Sectional-hyperbolic systems. Ergodic Theory and Dynamical System, 28:1587-1597, 2008.

[34] C. A. Morales, M. J. Pacifico, and E. R. Pujals. Robust transitive singular sets for 3-flows are partially hyperbolic attractors or repellers. Ann. of Math. (2), 160(2):375-432, 2004.

[35] M. J. Pacifico and M. Todd. Thermodynamic formalism for contracting Lorenz flows. Journal of Statistical Physics, 139(1):159-176, 2010.

[36] M. Pollicott. On the rate of mixing of Axiom A flows. Invent. Math., 81(3):413-426, 1985.

[37] M. Pollicott. Exponential mixing for the geodesic flow on hyperbolic three-manifolds. J. Statist. Phys., 67(3-4):667-673, 1992.

[38] M. Pollicott. On the mixing of Axiom A attracting flows and a conjecture of Ruelle. Ergodic Theory Dynam. Systems, 19(2):535-548, 1999.

[39] C. Pugh and M. Shub. Ergodic elements of ergodic actions. Compositio Math., 23:115-122, 1971.

[40] A. Renyi. Representations for real numbers and their ergodic properties. Acta Math. Acad. Sci. Hungar., 8:477-493, 1957.

[41] V. A. Rokhlin. On the fundamental ideas of measure theory. A. M. S. Transl., 10:1-52, 1962. Transl. from Mat. Sbornik 25 (1949), 107-150.

[42] A. Rovella. The dynamics of perturbations of the contracting Lorenz attractor. Bull. Braz. Math. Soc., 24(2):233-259, 1993.

[43] D. Ruelle. A measure associated with Axiom A attractors. Amer. J. Math., 98:619-654, 1976.

[44] D. Ruelle. Flots qui ne mélangent pas exponentiellement. C. R. Acad. Sci. Paris Sér. I Math., 296(4):191-193, 1983.

[45] Y. Sinai. Gibbs measures in ergodic theory. Russian Math. Surveys, 27:21-69, 1972.

[46] W. Tucker. The Lorenz attractor exists. C. R. Acad. Sci. Paris, 328, Série I:1197-1202, 1999.

[47] P. Varandas. Non-uniform specification and large deviations for weak Gibbs measures. ArXiv e-prints, June 2009.

Vítor Araújo, Departamento de Matemática, Universidade Federal da Bahia, Av. AdeMAR DE BARRos S/N, 40170-110 SAlVAdor, BRAZIL.

E-mail address: vitor.araujo@pq.cnpq.br

Paulo Varandas, Departamento de Matemática, Universidade Federal da Bahia, Av. Ademar de Barros s/n, 40170-110 Salvador, Brazil.

E-mail address: paulo.varandas@ufba.br 